M. NAGASE

KODAI MATH. J.

5 (198?), 38-64

\title{
DE RHAM-HODGE THEORY ON A MANIFOLD WITH CONE-LIKE SINGULARITIES
}

\author{
By MASAYoshi NAGASE
}

\section{Introduction}

The classical theorem of de Rham-Hodge asserts that the cohomology of an oriented closed Riemannian manifold can be represented by harmonic forms. The similar one still holds for an oriented compact Riemannian manifold with boundary by imposing certain boundary conditions, such as absolute and relative ones ([10]). But it is pointed out in [5] that, in genaral, such a result does not directly extend to non-compact cases, even if we use square-integrable forms. Nevertheless, there are analogues of the de Rham-Hodge theory on manifolds of certain types, such as an oriented Riemannian manifold with cylinders ([2]) and an oriented non-compact Riemannian manifold on which a discrete group $I$ of orientation-preserving isometries acts freely so that its quotient by $\Gamma$ is compact ([6]). These examples, however, are obtained from complete Riemannian manifolds.

Recently, it was announced by J. Cheeger ([4]) that the Hodge theory on compact (therefore, complete) Riemannian manifolds can be extended to incomplete Riemannian manifolds of certain types. Actually, according to his announcement, the $L^{2}$-cohomology group of an oriented Riemannian manifold $X=C_{0,1}(N)$ $\cup M$ with cone-like singularities is closely related to the space of harmonic forms on $X$ and the cohomology groups $H^{*}(M), H^{*}(M, N)$ and $i^{*}\left(H^{*}(M, N)\right.$ ), where the map $i$ is the inclusion $(M, \phi) \hookrightarrow(M, N)$, and, moreover, the Hodge theory itself still holds for $X$. He was further proceeding with saying, "Our results serve to illustrate new phenomena that are typical of the more general situation." Hence, we expect that, if we can find the other way to approach the Hodge theory on $X=C_{0,1}(N) \cup M$, such new phenomena will be clearer.

It seems that the motivation for Cheeger's work was aroused to solve the problem on analytic torsions which is known as Ray-Singer conjecture. This problem was independently solved by W. Müller ([9]) who made use of the simplicial approximation method which had been exploited by J. Dodziuk-V.K. Patodi ([7]). Therefore, we are naturally led to consider that there might be the way to reconstruct the de Rham-Hodge theory on $X=C_{0,1}(N) \cup M$ from the view-point of the simplicial approximation.

Then, our problem setting is now stated as follows. Is it possible to deduce

Received July 2, 1980 
Cheeger's results from the view-point of the simplicial $L^{2}$-cohomology? We especially want to establish a direct relation between the harmonic forms and the simplicial $L^{2}$-cohomology group. It will induce a relation between the harmonic forms and $H^{*}(M), H^{*}(M, N)$ and $i^{*}\left(H^{*}(M, N)\right)$ which agrees with Cheeger's results.

After explaining various notations and definitions in $\S 1$, we will state our results in $\S 2$.

The paper is devided into five sections as follows.

$\S 1$. Preliminaries

1.1. Spaces of differential forms

1.2. Simplicial $L^{2}$-cohomology

1.3. A manifold with cone-like singularities, its triangulation and its subdivisions

1.4. A manifold with cylinders, its triangulation and its subdivisions

1.5. De Rham map and Whitney map

1.6. $H_{(2)}^{p}\left(K^{*}\right)$ and $\bar{H}_{(2)}^{p}\left(K^{*}\right)$

1.7. Estimates on $C(N)$

1.8. Remark

$\S 2$. Main results

$\S 3$. The harmonic forms on $C(N)$; the proof of Theorem 2.2

$\S 4$. The proof of Theorem 2.1

§5. Appendix

The auther is grateful to Professor K. Shiga for giving him an opportunity of learning these subjects and for useful conversations during the preparation of the present paper.

\section{$\S 1$. Preliminaries}

1.1. Spaces of differential forms

Let $X$ be an oriented Riemannian manifold without boundary, which may be compact or not. But we are mainly concerned with a non-compact case. Let $\Lambda^{p}=\Lambda^{p}(X)$ be the space of smooth $p$-forms on $X$ and $\Lambda_{0}^{p}$ its subspace of compactly supported smooth $p$-forms on $X . \Lambda_{0}^{p}$ is a pre-Hilbert space with an inner product given by

$$
(\omega, \eta)_{0}=(\omega, \eta)_{0 ; X}=\int_{X} \omega \wedge * \eta^{(\dagger)},
$$

where $*$ is the $*$-operator associated with the Riemannian metric. The completion of $\Lambda_{0}^{p}$ with respect to this inner product is denoted by $\Lambda^{p: 0}$, which is regarded as a Hilbert space with this inner product.

( $\uparrow$ Since we consider $\bar{d}$, ( ) $)_{0}\|\|_{0}$ on various spaces, we will use subscripts to indicate the precise domains. For examples, $\bar{d}_{X},()_{0 ; X},\|\cdot\|_{0 ; X}$. 
Let $d_{p} ; \Lambda^{p} \rightarrow \Lambda^{p+1}$ be the exterior derivative, $\delta_{p}=(-1)^{p \operatorname{dim} X+\operatorname{dim} X+1} * d_{p} *$ the formal adjoint of $d_{p}$ and $\Delta_{p}=d_{p-1} \delta_{p-1}+\delta_{p} d_{p}$ the Laplacian.

To avoid the repetition occurring in defining the Sobolev spaces, we take a finite set $S$ which consists of polynomials generated by $d$ and $\delta$. As examples of $S$, we may take $\{d\},\{\delta\}$ or $\left\{\Delta, \Delta^{2}, \cdots \Delta^{k}\right\}$. First we put

$$
\Lambda_{S}^{p}=\left\{\omega \in \Lambda^{p ; 0} \cap \Lambda^{p} \mid \text { For any } D \in S, D \omega \text { is square-integrable. }\right\} .
$$

Then we define the Sobolev spaces $\Lambda^{p ; S}$ and $\bar{A}^{p ; S}$ associated with $S$ as follows.

Definition 1.1. $\Lambda^{p ; S}$ is the completion of $\Lambda_{S}^{p}$ by the norm

$$
\|\omega\|_{S}=\left(\|\omega\|_{0}^{2}+\sum_{D \in S}\|D \omega\|_{0}^{2}\right)^{1 / 2},
$$

where $\|\omega\|_{0}^{2}=(\omega, \omega)_{0}$. And $\bar{A}^{p ; S}$ is the closure of $\Lambda_{S}^{p}$ in $\Lambda^{p ; S}$.

In case $S=\phi$, we write $\|\omega\|_{0}$ for $\|\omega\|_{S}$, which coincides with the norm in $\Lambda^{p ; 0}$; hence we have ${ }^{p ; \phi}=\bar{\Lambda}^{p ; \dot{\phi}}=\bar{\Lambda}^{p ; 0}$. We prefer to use the notation $\Lambda^{p ; 0}$ in this case. Moreover we set

and

$$
\Lambda^{p ; k}=\Lambda^{\left.\left.p ;\{\lrcorner,\lrcorner^{2}, \cdots\right\lrcorner^{k}\right\}}, \quad \bar{\Lambda}^{p ; k}=\bar{\Lambda}^{p ;\left\{\Delta, \Delta^{2}, \cdots \Delta^{k}\right\}}
$$

$$
\|\omega\|_{k}=\|\omega\|_{\{\Delta, \Delta 2, \ldots \Delta k\}} .
$$

It is clear that the follwing implications of these spaces are valid.

$$
\begin{gathered}
\Lambda^{p} \supset \Lambda_{S}^{p} \supset \Lambda_{0}^{p} \\
\bigcap^{p ; 0} \supset \Lambda^{p ; S} \supset \widehat{\Lambda}^{p ; S}
\end{gathered}
$$

If $X$ is compact, we have $\Lambda^{p ; S}=\bar{\Lambda}^{p ; S}$. But, in case $X$ is non-compact, the situa-

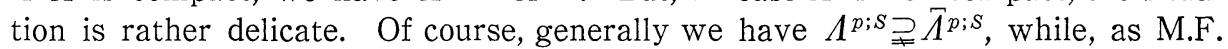
Atiyah ([1]) showed, if $X$ is a Galois-covering space of a compact manifold, we have $\Lambda^{\left.p ; i(I+\Delta)^{k}\right\}}=\bar{\Lambda}^{p ; i(I+\Delta)^{k_{1}}}$.

Besides, when we say a "Sobolev space", we usually regard it as a Hilbert space, but, in our case, we regard it just as a "Banach space", except the case $S=\phi$.

It is easily verified that $\Lambda^{p ; 0} \supset \Lambda_{\{d\}}^{p} \stackrel{d_{p}}{\longrightarrow} \Lambda^{p+1 ; 0}, \Lambda^{p ; 0} \supset \Lambda_{0}^{p} \stackrel{d_{p}}{\longrightarrow} \Lambda^{p+1 ; 0}, \quad \Lambda^{p+1 ; 0}$ $\supset \Lambda_{\{\delta\}}^{p+1 ; 0} \stackrel{\delta_{p}}{\longrightarrow} \Lambda^{p ; 0}$ and $\Lambda^{p+1 ; 0} \supset \Lambda_{0}^{p+1} \stackrel{\delta_{p}}{\longrightarrow} \Lambda^{p ; 0}$ are closable operators. Actually the domains of their respective closures $\bar{d}_{p}, \bar{d}_{p}, \bar{\delta}_{p}$ and $\bar{\delta}_{p}$ are biven by

and

$$
\operatorname{dom} \bar{d}_{p}=\Lambda^{p ;\{d\}}, \quad \operatorname{dom} \bar{d}_{p}=\bar{\Lambda}^{p ;\{d\}}, \quad \operatorname{dom} \bar{\delta}_{p}=\Lambda^{p+1 ;\{\delta\}}
$$

$$
\operatorname{dom} \hat{\delta}_{p}=\bar{\Lambda}^{p+1 ;\{\delta\}} \text {. }
$$

In general, if $X$ is non-compact, $\operatorname{dom} \bar{d}_{p} \supsetneq \operatorname{dom} \bar{d}_{p}$ and $\operatorname{dom} \bar{\delta}_{p} \supseteqq \operatorname{dom} \bar{\delta}_{p}$, moreover, for $\omega \in \Lambda^{p ; 0}, \Delta \omega=0$ (as a distribution) does not always imply $d \omega=\delta \omega=0$ (as dis- 
tributions). Therefore we should give clear definitions of what are called harmonic forms.

\section{Definition 1.2 .}

$$
\begin{aligned}
& \mathcal{H}^{p}(X)=\left\{\omega \in \Lambda^{p, 0} \mid d \omega=\delta \omega=0 \text { (as distributions) }\right\}, \\
& \overline{\mathcal{H}}_{d}^{p}(X)=\left\{\omega \in \operatorname{dom} \bar{d}_{p} \mid \tilde{d} \omega=0, \delta \omega=0 \text { (as a distribution) }\right\}, \\
& \overline{\mathcal{H}}_{\delta}^{p}(X)=\left\{\omega \in \operatorname{dom} \bar{\delta}_{p-1} \mid d \omega=0 \text { (as a distribution), } \delta \omega=0\right\} .
\end{aligned}
$$

By the well-known regularity theorem, we easily verify that

$$
\mathscr{H}^{p}(X)=\Lambda_{\{d, \hat{o}\}}^{p} \cap \mathcal{H}^{p}(X) .
$$

And we have

and

$$
\begin{aligned}
& \mathscr{H}^{p}(X)=\Lambda^{p ;\left\langle d, \delta_{1}\right.} \cap \mathcal{H}^{p}(X)=\operatorname{dom} \bar{d}_{p} \cap \operatorname{dom} \bar{\delta}_{p-11} \cap \mathcal{H}^{p}(X), \\
& \overline{\mathscr{H}}_{d}^{p}(X)=\operatorname{dom} \bar{d}_{p} \cap \mathcal{H}^{p}(X)
\end{aligned}
$$

$$
\overline{\mathcal{H}}_{S}^{p}(X)=\operatorname{dom} \bar{\delta}_{p-1} \cap \mathcal{H}^{p}(X) .
$$

LEMMA 1.3.

(1) $\operatorname{ker} \bar{d}_{p}=\mathscr{H}^{p}(X) \oplus \overline{d \Lambda_{0}^{p-1}\left({ }^{\dagger}\right)}$,

(2) $\overline{\operatorname{im} \hat{d}_{p-1}}=\overline{d \Lambda_{0}^{p-1}}$,

(3) $\operatorname{ker} \tilde{d}_{p}=\overline{\mathscr{H}}_{d}^{p}(X) \oplus \overline{\operatorname{im} \tilde{d}_{p-1}}$. $([5])$.

Proof. The space $A^{p ; 0}$ has the following orthogonal Hodge decomposition

$$
\Lambda^{p, 0}=\mathscr{M}^{p}(X) \oplus \overline{d \Lambda_{0}^{p-1}} \oplus \overline{\delta \Lambda_{0}^{p+1}} .
$$

The lemma is then a natural consequence of this decomposition.

On the other hand, according to M. F. Atiyah ([1]), we call $\bar{\Lambda}^{p ; k}=\bar{\Lambda}^{\left.p ;:(I+\Delta)^{k}\right\}}$ and $\Lambda^{p ;\left(\langle I+\Delta)^{k}\right\}}$ the minimal and maximal domains of $(I+\Delta)^{k}$. If these domains coincide, then $\Lambda^{p ; i(I+\Delta)^{k_{1}}}=\Lambda^{p ; k}$ as Banach spaces. In this case, we regard $\Lambda^{p ; k}$ as a Hilbert space with the natural inner product given by

$$
(\omega, \eta)_{k}=\left((I+\Delta)^{k} \omega,(I+\Delta)^{k} \eta\right)_{0}
$$

for $\omega, \eta \in \Lambda^{p ; k}=\Lambda^{p,\{I+\perp)^{k_{1}}}=\bar{\Lambda}^{p ;(I+\Delta)^{k_{l}}}=\bar{\Lambda}^{p ; k}$.

Then the following proposition follows from the same proof as that of [1] and [6].

Proposition 1.4. If the minmal and maximal domains of $(I+\Delta)^{k}$ corncide

(†) Unless otherwise stated, the "closure" operation is always taken in the $L^{2}$-sense. 
for $k>0,(I+\Delta)^{k}$ is a self-adjoint operator on $\Lambda^{p ; 0}$ with domain $\Lambda^{p ; k}$ and a Hilbert space isomorphism of $\Lambda^{p ; k}$ onto $\Lambda^{p ; 0}$, moreover, we have

(1) $\mathscr{\mathcal { H }}^{p}(X)=\overline{\mathscr{H}}_{d}^{p}(X)=\overline{\mathcal{H}}_{\tilde{\delta}}^{p}(X)$

$$
\begin{aligned}
& =\left\{\omega \in \Lambda^{p ; 0} \mid \Delta \omega=0 \text { (as a distribution) }\right\} \\
& =\left\{\omega \in \Lambda^{p ; l} \mid \bar{d} \omega=\bar{\delta} \omega=0\right\}=\left\{\omega \in \Lambda^{p ; l} \mid \Delta \omega=0\right\}
\end{aligned}
$$

for $l=1,2, \cdots$,

(2) $\overline{\operatorname{im} \bar{d}_{p-1}}=\overline{d \Lambda_{0}^{p-1}}$.

\subsection{Simplicial $L^{2}$-cohomology}

We define the simplicial $L^{2}$-cohomology spaces of an infinite simplicial complex $K$ of which each $p$-simplex is a face of at most $n$ simplexes of dimension $p+1$, where the integer $n$ is independent of simplexes. Let $C^{p}(K)$ be the space of real, oriented cochains of $K$ of degree $p$. We think of cochains $f \in C^{p}(K)$ as infinite formal linear combinations of oriented simplexes of $K$. A cochain $f=\sum f_{\sigma} \cdot \sigma$ is in $L^{2}, f \in C_{2}^{p}(K)$, if the sum of squares of coefficients is finite. Since there exists the above integer $n$, the simplicial coboundary $d_{c} f$ of an $L^{2}$ cochain $f$ is in $L^{2}$ and the coboundary operator $d_{c}: C_{2}^{p}(K) \rightarrow C_{2}^{p+1}(K)$ is bounded. We define the simplicial $L^{2}$-cohomology spaces as follows.

$$
H_{(2)}^{p}(K)=\operatorname{ker} d_{c, p} / \operatorname{im} d_{c, p-1} \quad \text { and } \quad \bar{H}_{(2)}^{p}(K)=\operatorname{ker} d_{c, p} / \overline{\operatorname{im} d_{c, p-1}},
$$

where $\overline{1 \mathrm{~m} d_{c, p-1}}$ denotes the closure of im $d_{c, p-1}$ with respect to the norm

$$
\|f\|\left(\sum f_{\sigma}^{2}\right)^{1 / 2} \quad \text { for } f=\sum f_{\sigma} \cdot \sigma \in C_{2}^{p}(K) .
$$

\subsection{A manifold with cone-like singularities, its triangulation and its subdivisions}

Let $N$ be an $m$-dimensional oriented closed Riemannian manifold (which might not be connected) with metric $g$. By the metruc cone $C_{0, u}(N)$ on $N$, we mean the space $(0, u] \times N$, equipped with the metric

$$
d r \otimes d r+r^{2} g .
$$

Definition 1.5. An $(m+1)$-dimensional oriented Riemannian manifold $X$ without boundary is said to have cone-like singularities if there exists an $(m+1)$ dimensional compact submanifold $M$ in $X$ such that $X \backslash$ int $M$ is isometric to a disjoint union $\bigcup_{\jmath=1}^{k} C_{0, u_{j}}\left(N_{\jmath}^{m}\right)$ of a finite number of metric cones for some $u_{\jmath}>0$ and $N_{\jmath}^{m}$.

It is easily verified that, without losing generality, we may assume $k=1$ and $u_{1}=1$. And we write $X=C_{0,1}(N) \cup M$, where $\partial M=N$ and the union is along the boundary. Therefore, $X$ is an incomplete Riemannian manifold. 


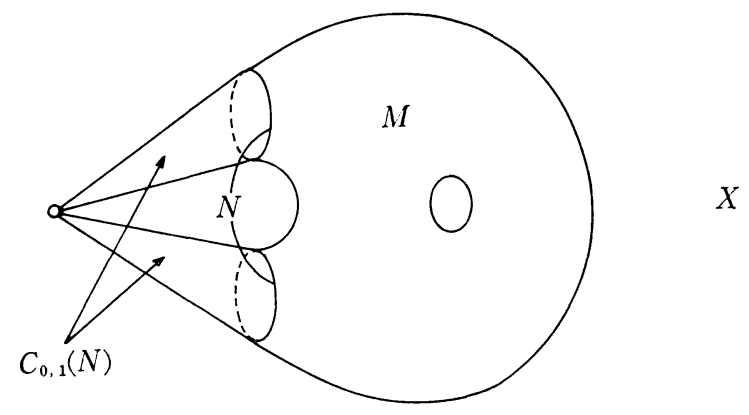

Now we triangulate $X$ in such a way that its infinite simplicial complex $K^{*}$ satisfies the condition in $\mathbf{1 . 2}$ and is available for our purposes.

Let $\left\{\left[\eta^{n+1}, \eta^{n}\right]\right\}_{n=0}^{\infty}$ be a triangulation of $(0,1]$, where $\eta$ is a fixed real number such as $0<\eta<1$. Put $N_{n}=C_{\eta^{n+1, \eta n}}(N)=\left[\eta^{n+1}, \eta^{n}\right] \times N$ with metric $d r \otimes d r$ $+r^{2} g$, and $\pi_{n}: C_{0,1}(N) \ni(r, x) \mapsto\left(\eta^{n} r, x\right) \in C_{0,1}(N)$.

Let $(K, L)$ be an arbitrary triangulation of $(M, N)$. Then we have a cellular decomposition $\{[\eta,(1+\eta) / 2] \times \sigma,[(1+\eta) / 2,1] \times \sigma, \eta \times \sigma,(1+\eta) / 2 \times \sigma, 1 \times \sigma\}_{\sigma \in L}$ of $N_{0}$. We subdivide it into a simplicial complex $L_{0}$ without adding new vertices and symmetrically with respect to the face $(1+\eta) / 2 \times N$. The triangulation of $N_{n}$ is given by $L_{n}=\pi_{n}\left(L_{0}\right)$. The collection $K^{*}$ of all these simplicial complexes forms a triangulation of $X$.

Next we describe a method of subdividing $K^{*}$. The method is an application of the standard subdivision introduced by $\mathrm{H}$. Whitney in [11]. It is very well suited to our purposes. Let $\left\{p_{1}, \cdots, p_{l}\right\}$ be all vertices of $L$ and $\left\{q_{1}, \cdots, q_{k}\right\}$ all vertices of $K \backslash L$. We give the following ordering to vertices of $K \cup L_{0}$.

$$
\begin{aligned}
& \frac{1+\eta}{2} \times p_{1}<\frac{1+\eta}{2} \times p_{2}<\cdots \cdots \cdots \cdots<\frac{1+\eta}{2} \times p_{l} \\
& <\eta \times p_{1}<\eta \times p_{2}<\cdots \cdots \cdots \cdots \cdots \cdots \cdots \cdots \cdots+\cdots \cdots+\cdots \cdots \\
& <1 \times p_{1}=p_{1}<1 \times p_{2}=p_{2}<\cdots \cdots \cdots \cdots 1 \times p_{l}=p_{l} \\
& <q_{1}<q_{2}<\ldots \ldots \ldots \ldots<q_{k}
\end{aligned}
$$

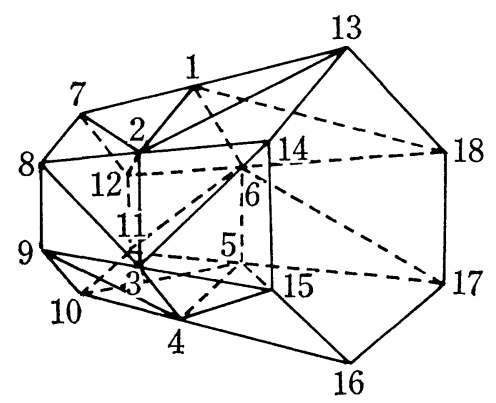


This ordering gives us the standard subdivision $S d\left(K \cup L_{0}\right)$ of $K \cup L_{0}([11])$. Each simplex of $S d\left(K \cup L_{0}\right)$ has ordered vertices and we can subdivide it again. Inductively, we define

$$
S d^{k+1}\left(K \cup L_{0}\right)=S d\left(S d^{k}\left(K \cup L_{0}\right)\right) .
$$

Then $S d^{k+1}\left(L_{n}\right)=\pi_{n}\left(S d^{k+1}\left(L_{0}\right)\right)$ is a subdivision of $S d^{k}\left(L_{n}\right)$. Put

and

$$
S d^{k}\left(C_{0,1}(L)\right)=C_{0,1}\left(S d^{k}(L)\right)=S d^{k}\left(L_{0}\right) \cup S d^{k}\left(L_{1}\right) \cup \cdots
$$

$$
S d^{k}\left(K^{*}\right)=S d^{k}(K) \cup S d^{k}\left(C_{0,1}(L)\right) \text {. }
$$

Then $S d^{k}\left(K^{*}\right)$ is a subdivision of $K^{*}, k=0,1,2, \cdots$.

\subsection{A manifold with cylinders, its triangulation and its subdivisions}

Let $M$ and $N=\partial M$ be the same Riemannian manifolds as in 1.3. We suitably change the metric on $M$ near $N$ in such a way that there exists a neighborhood of $N$ which is isometric to $[0, \varepsilon) \times N$ with metric $d u \otimes d u+g$ for some $\varepsilon>0$. The manifold with this changed metric is also denoted by the same symbol $M$. Set $X^{\prime}=(-\infty, 0] \times N \cup M$ (the union is along the boundary), which we call a manifold with cylinders.

Let $(K, L)$ be the triangulation of $(M, N)$ used in 1.3, which we also assume a triangulation of $(M, N)$ of this case. The triangulation $K^{* \prime}$ of $X^{\prime}$ is given by the similar way as in 1.3. The notations in this case are similarly defined.

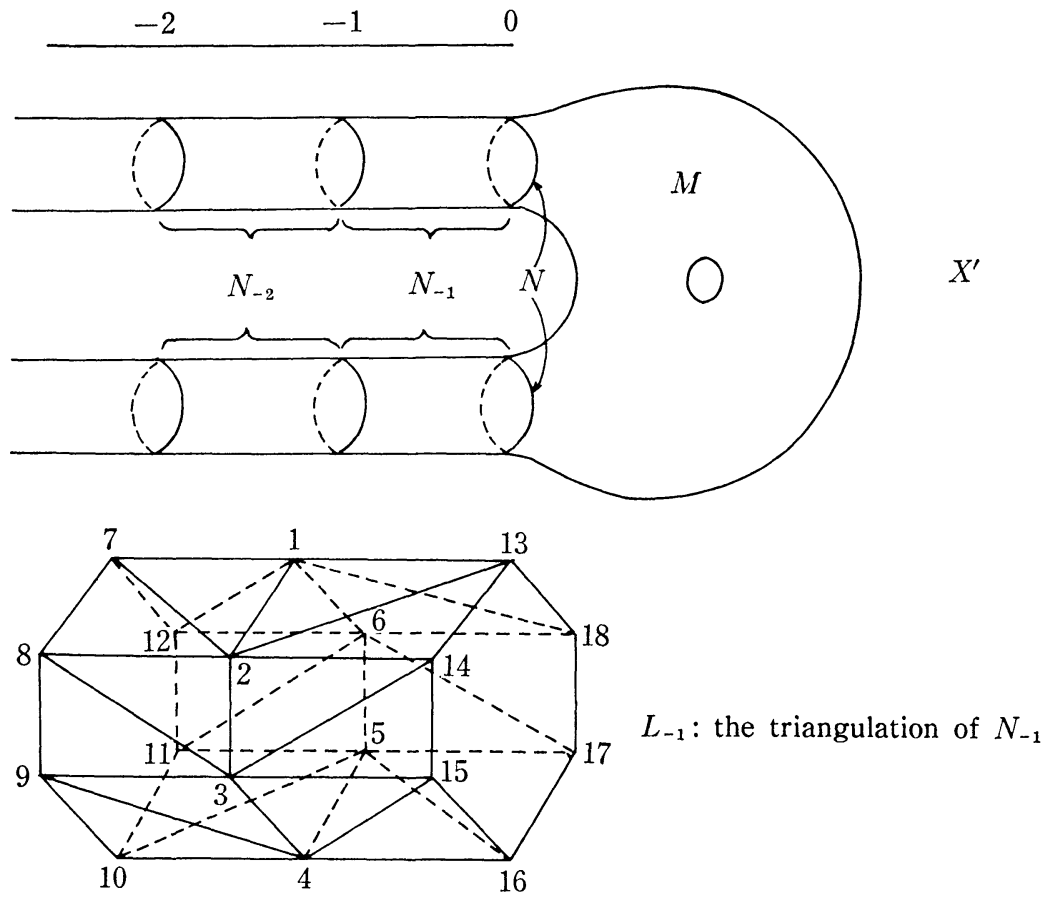




$$
\begin{gathered}
\pi_{n}^{\prime}:(-\infty, \infty) \times N \longrightarrow(-\infty, \infty) \times N, \text { for } n=0,-1,-2, \cdots, \\
\Psi \\
(u, x) \longmapsto(u+n, x)
\end{gathered}
$$

$L_{n}=\pi_{n-1}^{\prime}\left(L_{-1}\right)$; the triangulation of $N_{n}$, $K^{* \prime}=K \cup L_{-1} \cup L_{-2} \cup \cdots$; the triangulation of $X^{\prime}$, $S d^{k}\left(K^{* \prime}\right)$.

Remark. $K^{*}=K^{* \prime}, S d^{k}\left(K^{*}\right)=S d^{k}\left(K^{* \prime}\right)$ as simplicial complexes ; hence, when we treat these simplicial complexes, we prefer to use the notations in 1.3.

\subsection{De Rham map and Whitney map}

We continue to use the same notations as in 1.3 and 1.4 .

The de Rham map $\int: \Lambda^{p}(X) \rightarrow C^{p}\left(K^{*}\right)$ is defined by $\int \omega=\sum_{\sigma}\left(\int_{\sigma} \omega\right) \cdot \sigma$, where $\sigma$ runs over all $p$-simplexes of $K^{*}$. The de Rham map $\int^{\prime}: \Lambda^{p}\left(X^{\prime}\right) \rightarrow C^{p}\left(K^{*}\right)$ is also similarly defined.

Next, according to $\mathrm{H}$. Whitney, we define the Whitney map $W: C^{p}\left(K^{*}\right) \rightarrow$ $\Lambda^{p}(X)$ in the following way. Let $\left\{U_{v}\right\}_{v \in\left(K^{*}\right)^{0}}$ be an open covering of $X$ by open stars of vertices of $K^{*},\left\{\varphi_{v}\right\}_{v \in\left(K^{*}\right)^{0}}$ be a $C^{\infty}$-partition of unity on $X$ subordinate to $\left\{U_{v}\right\}$ such that $\varphi_{v} \cdot \pi_{n}=\varphi_{\pi_{-n}(v)}$ for $v \in\left(C_{0,1}(L) \backslash L\right)^{0}$. If $\sigma=\left[v_{0}, \cdots, v_{p}\right]$ is an oriented $p$-simplex of $K^{*}$, then

$$
W \sigma= \begin{cases}\varphi_{v_{0}} & : p=0, \\ p ! \sum_{i=0}^{p}(-1)^{2} \varphi_{v_{i}} d \varphi_{v_{\imath}} \wedge \cdots \wedge \widehat{d \varphi_{v_{i}}} \wedge \cdots \wedge d \varphi_{v_{p}}: p=0 .\end{cases}
$$

Now, for an arbitrary cochain $f=\sum f_{\sigma} \cdot \sigma$, we define $W f=\sum f_{\sigma} \cdot W \sigma$. As supp $\varphi_{v}$ $\subset \overline{s t \sigma}$, where $v$ is a vertex of $\sigma$, this sum is locally finite and defines a $C^{\infty}$-form. The Whitney map has the following properties.

(1) $d \circ W=W \circ d_{c}$,

(2) $\int \circ W=I$ on $C^{*}\left(K^{*}\right)$,

(3.) $\pi_{n}^{*} W f=W\left(\pi_{-n}(f)\right)$.

These properties are proved in [11] and by our choice of partition of unity. If necessary, we use the partition of unity by the barycentric coordinate functions to construct the Whitney map. In this case, (1), (2) and (3) remain true, but the image of $W$ no longer consists of $C^{\infty}$-forms.

The Whatney map $W^{\prime}: C^{p}\left(K^{*}\right) \rightarrow \Lambda^{p}\left(X^{\prime}\right)$ is similarly defined and has the same properties.

1.6. $H_{(2)}^{p}\left(K^{*}\right)$ and $\bar{H}_{(2)}^{p}\left(K^{*}\right)$

We continue to use the same notations as in $\mathbf{1 . 3}$.

Since $K^{*}$ and $S d^{k}\left(K^{*}\right)$ satisfy the condition of 1.2 , we can define the $L^{2}$ - 
cohomology spaces $H_{(2)}^{p}\left(K^{*}\right), \bar{H}_{(2)}^{p}\left(K^{*}\right), H_{(2)}^{p}\left(S d^{k}\left(K^{*}\right)\right), \bar{H}_{(2)}^{p}\left(S d^{k}\left(K^{*}\right)\right)$. First we study the basic properties of the natural subdivision map.

Proposition 1.6. The subdivision map $s: C^{p}\left(S d\left(K^{*}\right)\right) \rightarrow C^{p}\left(K^{*}\right)$ restricts to a bounded operator $s: C_{2}^{p}\left(S d\left(K^{*}\right)\right) \rightarrow C_{2}^{p}\left(K^{*}\right)$ and induces isomorphisms;

$$
H_{(2)}^{p}\left(S d\left(K^{*}\right)\right) \stackrel{\simeq}{\leftrightharpoons} H_{(2)}^{p}\left(K^{*}\right), \quad \bar{H}_{(2)}^{p}\left(S d\left(K^{*}\right)\right) \cong \bar{H}_{(2)}^{p}\left(K^{*}\right) .
$$

Proof. Note that $s$ is a cochain map and a local operator. More precisely, for every $p$-simplex $\sigma$ of $S d\left(K^{*}\right), s(\sigma)=\tau$, where $\tau$ is the unique $p$-simplex of $K^{*}$ such that Int $|\sigma| \subset \operatorname{Int}|\tau|$. As a result, it is easily verified that $s$ can restrict to a bounded operator and induce the maps $\tilde{s}: H_{(2)}^{p}\left(S d\left(K^{*}\right)\right) \rightarrow H_{(2)}^{p}\left(K^{*}\right)$ and $\tilde{s}: \bar{H}_{(2)}\left(S d\left(K^{*}\right)\right) \rightarrow \bar{H}_{(2)}^{p}\left(K^{*}\right)$. To prove that these are isomorphisms, it is sufficient to show the existence of a cochain map $t: C^{p}\left(K^{*}\right) \rightarrow C^{p}\left(S d\left(K^{*}\right)\right)$ with the following properties.

(i) $s \circ t=I$ on $C^{p}\left(K^{*}\right)$.

(ii) $t$ is local in the sense that, for every $p$-simplex $\tau$ of $K^{*}, t(\tau)=\sigma$ for some $p$-simplex $\sigma$ such that $|\sigma| \subset|\tau|$.

(iii) $t_{\circ} S$ is cochain-homotopic to the identity on $C^{*}\left(S d\left(K^{*}\right)\right)$, i.e. there exists a family of maps $G: C^{*}\left(S d\left(K^{*}\right)\right) \rightarrow C^{*-1}\left(S d\left(K^{*}\right)\right)$ such that

$$
t \circ s-I=G \circ d_{c}+d_{c} \circ G \text {. }
$$

Moreover $G$ is local in the sense that, for every simplex $\sigma$ of $S d\left(K^{*}\right)$, the cochain $G(\sigma)$ is supported in the uniquely determined closed simplex of $K^{*}$ whose interior contains Int $|\sigma|$.

These maps can be constructed in the following way. Construct $t^{\prime}, G^{\prime}$ satisfying (i), (ii) and (iii) on $K \cup L_{-1}$ and $S d(K) \cup S d\left(L_{-1}\right)$. Then define the following operation; turn down $[-1,-1 / 2] \times L$ on $[-3 / 2,-1] \times L$ then $[-3 / 2,-1]$ $\times L$ on $[-2,-3 / 2] \times L$ and so on.

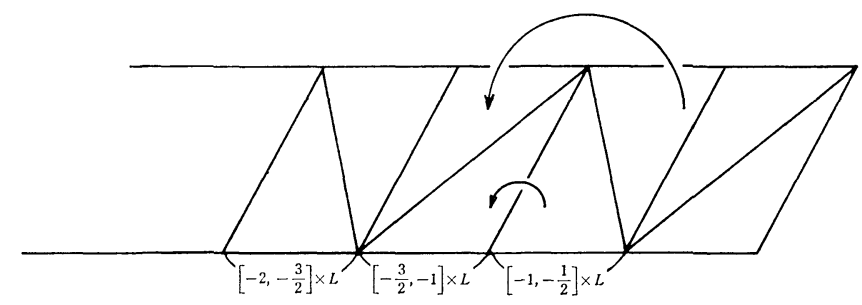

If an oriented simplex $\sigma$ of $[-1,-1 / 2] \times L$ is carried to an oriented simplex $\tau$ by this operation, where the orientations must be turned down accordingly, then we define that $t(\tau)$ and $G(\tau)$ are the images of $t^{\prime}(\sigma)$ and $G^{\prime}(\sigma)$ by the operation, respectively. Then the proof that $t$ is a cochain map and satisfies the three properties is straight-forward. 
Next, since there exist the short exact sequences of cochain complexes

$$
\begin{aligned}
& 0 \longrightarrow C^{*}(K, L) \stackrel{\imath_{1}}{\longrightarrow} C^{*}(K) \stackrel{\jmath_{1}}{\longrightarrow} C^{*}(L) \longrightarrow 0 \\
& 0 \longrightarrow C^{*}(K, L) \stackrel{\imath_{2}}{\longrightarrow} C_{2}^{*}\left(K^{*}\right) \stackrel{j_{2}}{\longrightarrow} C_{2}^{*}\left(C_{0,1}(L)\right) \longrightarrow 0,
\end{aligned}
$$

we have the following long exact sequences.

$$
\begin{aligned}
& \cdots \rightarrow H^{p-1}(L) \stackrel{\delta_{1}}{\longrightarrow} H^{p}(K, L) \stackrel{\imath_{1}}{\longrightarrow} H^{p}(K) \stackrel{\jmath_{1}}{\longrightarrow} H^{p}(L) \rightarrow \cdots \\
& \cdots \rightarrow H_{(2)}^{p-1}\left(C_{0,1}(L)\right) \stackrel{\delta_{2}}{\longrightarrow} H^{p}(K, L) \stackrel{\iota_{2}}{\longrightarrow} H_{(2)}^{p}\left(K^{*}\right) \stackrel{j_{2}}{\longrightarrow} H_{(2)}^{p}\left(C_{0,1}(L)\right) \rightarrow \cdots
\end{aligned}
$$

PROPOSITION 1.7.

(1) $H_{(2)}^{p}\left(K^{*}\right) \cong i_{2}\left(H^{p}(K, L)\right) \oplus H_{(2)}^{p}\left(C_{0,1}(L)\right)$,

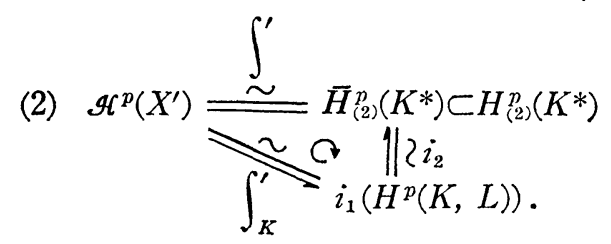

Proof. Let $S$ be a natural triangulation of the cylinder $(-\infty, \infty) \times N$.

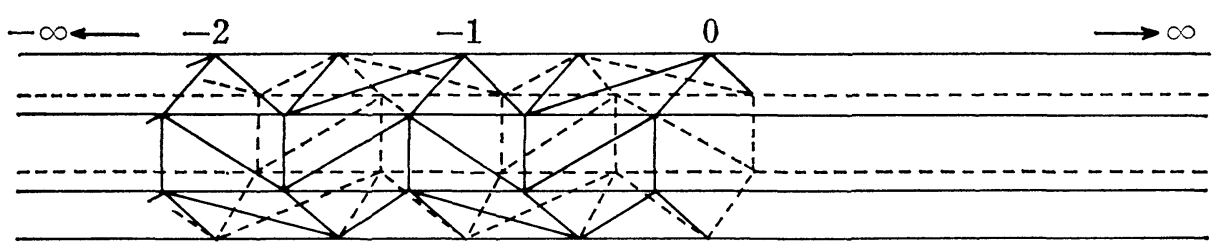

Then $\bar{H}_{(2)}^{p}(S) \stackrel{\mathcal{J}}{\cong} \mathcal{H}^{p}((-\infty, \infty) \times N) \stackrel{\int_{[-1,0] \times N}}{\cong} \imath_{1}\left(H^{p}([-1,0] \times N,\{-1\} \times N \cup\{0\} \times N)\right)$

Therefore we have

$$
\cong 0 \quad([2],[6]) \text {. }
$$

$$
\operatorname{ker} d_{c, s}=\overline{\operatorname{im} d_{c, s}} \text {. }
$$

For $f \in \bar{H}_{(2)}^{p}\left(K^{*}\right)$, we put $g=f_{1 C_{0,1}(L)} \cup f_{1 C_{0,1}(L)}$. Then $g \in \operatorname{ker} d_{c, s}$. Therefore, (1.3) implies

$$
f_{1\{1\} \times L}=g_{1\{1\} \times L} \in d_{c, L}\left(C^{p-1}(L)\right) \text {. }
$$

Consequently the image of the map $\bar{H}_{(2)}^{p}\left(K^{*}\right) \ni[f] \stackrel{\imath}{\longmapsto}\left[f_{\mid K}\right] \in H^{p}(K)$ is contained in $i_{1}\left(H^{p}(K, L)\right)$ and $\imath$ is the inverse map of $\imath_{2}: \imath_{1}\left(H^{p}(K, L)\right) \rightarrow \bar{H}_{(2)}^{p}\left(K^{*}\right)$. Therefore $\iota_{2}: i_{1}\left(H^{p}(K, L)\right) \rightarrow \bar{H}_{(2)}^{p}\left(K^{*}\right)$ is isomorphic. Since $\int_{K}^{\prime}$ is isomorphic ([2]), in 
order to prove (2), it suffices to show that the image of $\int^{\prime}$ is contained in $C_{2}^{p}\left(K^{*}\right)$, which is samely proved as [6]. (Since the minimal and maximal domains of $(I+\Delta)^{k}$ on $X^{\prime}$ coincide, it is directly proved that $\int$ is isomorphic. (Proposition 1.4 and $[6]))$.

Next, in order to prove (1), we will show that $\delta_{2}$ is the 0 -map. For $[f] \in$ $H_{(2)}^{p-1}\left(C_{0,1}(L)\right)$, (1.3) implies that there exists $h \in C^{p-2}(L)$ such that $f_{\mid\{1\} \times L}=d_{c, L} h$. Therefore $g=\left(f-d_{c, C_{0,1}(L)} h\right)+d_{c} h$ belongs to ker $d_{c, K^{*}}$ and $g_{C_{0,1}(L)}=f$, which imply $\delta_{2}[f]=0$.

\subsection{Estimates on $C(N)$}

Let $C(N)$ be $(0, \infty) \times N$ equipped with the metric $d r \otimes d r+r^{2} g$. For an arbitrary smooth $p$-form $\omega(r, x)=\omega_{1}(r, x)+d r \wedge \omega_{2}(r, x)$ on $C(N)$, where $\omega_{1}$ and $\omega_{2}$ are $p$ and $(p-1)$-forms on $N$ smoothly parametrized by $r \in(0, \infty)$, respectively, we define several norms as follows.

Definition 1.8. For $r, s>0$, we put

$$
\|\omega\|_{(s, r)}=\left\{\int_{(S, N)}\left[\left(\iota_{s, r}^{*} \omega_{1}\right)(s, x) \wedge \widetilde{*}_{s}\left(\iota_{s, r}^{*} \omega_{1}\right)(s, x)+\left(\iota_{s, r}^{*} \omega_{2}\right)(s, x) \wedge \widetilde{*}_{s}\left(\iota_{s, r}^{*} \omega_{2}\right)(s, x)\right]\right\}^{1 / 2},
$$

where $\iota_{s, r}:(s, N) \ni(s, x) \rightarrow(r, x) \in(r, N)$ and $\widetilde{*}_{s}$ is the $*$-operator on $(s, N)$ whose metric is $s^{2} g$. Moreover, we put

and

$$
\|\omega\|_{0 ; r_{1}, r_{2}}=\left\{\int_{C_{r_{1}, r_{2}(N)}} \omega(r, x) \wedge * \omega(r, x)\right\}^{1 / 2}
$$

$$
\|\omega\|_{k ; r_{1}, r_{2}}=\left\{\sum_{0 \leqq l \leqq k}\left\|\Delta^{l} \omega\right\|_{0 ; r_{1}, r_{2}}^{2}\right\}^{1 / 2}
$$

for $0 \leqq r_{1}<r_{2}$.

The definition of the norm $\|\omega\|_{(s, r)}$ is essentially due to J. Cheeger ([3], [4]).

Then we have

and

$$
\begin{aligned}
& \|\omega\|_{0 ; r_{1}, r_{2}}^{2}=\int_{r_{1}}^{r_{2}}\|\omega\|_{(r, r)}^{2} d r=\int_{r_{1}}^{r_{2}}\left\{\left\|\omega_{1}\right\|_{(r r)}^{2}+\left\|\omega_{2}\right\|_{1}^{2}(r, r)\right\} d r, \\
& \left\|\omega_{1}\right\|_{(r, r)}^{2}=r^{m-2 p}\left\|\omega_{1}\right\|_{(1, r)}^{2}
\end{aligned}
$$

Therefore, we have

$$
\left\|\pi_{n}^{*} \omega_{1}\right\|_{(1, r)}^{2}=\left\|\omega_{1}\right\|_{(1, \eta n}^{2} n_{r} .
$$

$$
\left\|\pi_{n}^{*} \omega_{1}\right\|_{0 ; \eta, 1}^{2}=\int_{\eta}^{1} r^{m-2 p}\left\|\omega_{1}\right\|_{(1, \eta n}^{2} d r=\eta^{-n(m-2 p+1)}\left\|\omega_{1}\right\|_{0 ; \eta n+1, \eta n}^{2}
$$

and

$$
\left\|\pi_{n}^{*} \omega\right\|_{0 ; \eta, 1}^{2}=\left\|\pi_{n}^{*} \omega_{1}\right\|_{0 ; \eta, 1}^{2}+\eta^{2 n}\left\|\pi_{n}^{*} \omega_{2}\right\|_{0 ; \eta, 1}^{2}=\eta^{-n(m-2 p+1)}\|\omega\|_{0 ; \eta, n+1, \eta n}^{2} .
$$

Moreover, by using volume elements, we can easily prove 
Then we have

$$
\iota_{1, r}^{*} \widetilde{*}_{r} \omega_{1}=r^{m-2 p} \widetilde{*}_{1} \iota_{1, r}^{*} \omega_{1} .
$$

$$
\left(\pi_{n}^{*} \widetilde{*} \omega_{1}\right)(r, x)=\left(\iota_{r, \eta}^{*} n_{r} \widetilde{*}_{\eta} n_{r} \omega_{1}\right)(r, x)=\eta^{n(m-2 p)}\left(\widetilde{*}_{r} \pi_{n}^{*} \omega_{1}\right)(r, x)
$$

and

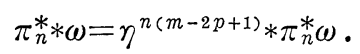

Therefore we have

and

$$
|\omega(r, x)|=\eta^{-p n}\left|\left(\pi_{n}^{*} \omega\right)\left(\eta^{-n} r, x\right)\right|
$$

$$
\pi_{n}^{*} \Delta \omega=\eta^{-2 n} \Delta \pi_{n}^{*} \omega
$$

We also define $\|\omega\|_{k ; U}$ by the formula

$$
\|\omega\|_{k ; U}=\left(\int_{U} \sum_{0 \leq l \leqq k} \Delta^{l} \omega \wedge * \Delta^{l} \omega\right)^{1 / 2}
$$

for every relatively compact, open subset $U \subset C(N)$ and every smooth $p$-form $\omega$ on $C(N)$. Then (1.6) implies

$$
\left\|\pi_{n}^{*} \omega\right\|_{0 ; U}^{2}=\eta^{-n(m-2 p+1)}\|\omega\|_{0 ; \pi n}^{2}(U) .
$$

Proposition 1.9. The de Rham map $\int$ induces a bounded operator

$$
\int: \Lambda^{p ; k}\left(C_{0,1}(N)\right) \longrightarrow C_{2}^{p}\left(C_{0,1}(L)\right)
$$

for $2 p \geqq m+1$ and $k>(m+1) / 4+1 / 2$.

Proof. Every element of $A^{p ; k}\left(C_{0,1}(N)\right)$ is of class $C^{1}$. Let $\tau_{1}, \cdots, \tau_{l}$ be all $p$-simplexes of $L_{0} \backslash\{\eta\} \times L$ and $U_{1}, \cdots, U_{l}$ their relatively compact neighborhoods, respectively. Then, for $\omega \in \Lambda^{p ; k}\left(C_{0,1}(N)\right)$,

$$
\begin{aligned}
\left(\int_{\pi_{n}\left(\tau_{j}\right)} \omega\right)^{2} & =\left(\int_{\tau_{j}} \pi_{n}^{*} \omega\right)^{2} \\
& \leqq\left(\operatorname{vol} \tau_{\jmath}\right)^{2}\left(\sup _{y \in \tau_{j}}\left|\left(\pi_{n}^{*} \omega\right)(y)\right|\right)^{2} \\
& \leqq\left(\operatorname{vol} \tau_{\jmath}\right)^{2} \cdot C \cdot\left\|\pi_{n}^{*} \omega\right\|_{k ; U_{\jmath}}^{2} \quad \text { (Sovolev lemma) } \\
& \leqq C\left(\operatorname{vol} \tau_{j}\right)^{2} \sum_{0 \leqq l \leqq k}\left\|\pi_{n}^{*} \Delta^{l} \omega\right\|_{0 ; U_{\jmath}}^{2} \\
& \left.\leqq C\left(\operatorname{vol} \tau_{\jmath}\right)^{2} \eta^{-n(m-2 p+1)}\|\omega\|_{k ; \pi n}^{2}\left(U_{j}\right), \quad \text { (by (1.9) and }(1.10)\right)
\end{aligned}
$$

where $C>0$ is independent of $n, \jmath$ and $\omega$. Therefore, since $m-2 p+1 \leqq 0$, we have

$$
\Sigma\left(\int_{\sigma} \omega\right)^{2} \leqq C^{\prime}\|\omega\|_{k}^{2}
$$


where $C^{\prime}>0$ is independent of $\omega$.

Therefore, we have

COROLLARY 1.10. The de Rham map $\int$ induces a bounded operator

$$
\int: \Lambda^{p ; k}(X) \longrightarrow C_{2}^{p}\left(K^{*}\right)
$$

for $X$ defined in $1.3,2 p \geqq m+1$ and $k>(m+1) / 4+1 / 2$.

\subsection{Remark}

Unless otherwise specified in the subsequent sections, $X$ and $K^{*}$ denote the manifold with cone-like singularities and its triangulation defined in $\mathbf{1 . 3}$.

\section{$\S 2$. Main results}

We can now describe the main results.

\section{THEOREM 2.1.}

(2.1) In case $2 p>m+1$, we have an isomorphism;

$$
\int: \mathscr{H}^{p}(X) \stackrel{\sim}{\simeq} \imath_{2}\left(H^{p}(K, L)\right) .
$$

(2.2) In case $2 p=m+1$, we have an isomorphism;

$$
\int: \mathscr{H}^{p}(X) \stackrel{\sim}{\rightleftharpoons} i_{2}\left(i_{1}\left(H^{p}(K, L)\right)\right)
$$

(2.3) In case $2 p<m+1$, for some $\omega \in \mathscr{H}^{p}(X), \int \omega$ might not be an $L^{2}$-cochain.

Remark. The following implications are valid; see Proposition 1.7.

$$
\begin{aligned}
& \imath_{2}\left(i_{1}\left(H^{p}(K, L)\right)\right) \subset \imath_{2}\left(H^{p}(K, L)\right) \subset H_{(2)}^{p}\left(K^{*}\right) \\
& \| \\
& \bar{H}_{(2)}^{p}\left(K^{*}\right) .
\end{aligned}
$$

THEOREM 2.2. The restriction to $C_{0,1}(N)$ of any element of $\mathcal{H}^{p}(X)$ can be written as a convergent sum of forms of the following types;

(2.4) in case $2 p>m+1, d\left(r^{2 \alpha p-1} \psi\right)$ and $d\left(r^{\alpha p-1+\sqrt{\alpha_{p-1}^{2}+\mu}} \omega_{\mu}\right)$,

(2.5) in case $2 p=m+1, d\left(r^{\sqrt{\mu}} \omega_{\mu}\right)$ and

(2.6) in case $2 p<m+1, \phi$ and $d\left(r^{\alpha p-1+\sqrt{\alpha_{p-1}^{2}+\mu}} \omega_{\mu}\right)$,

where $\phi$ and $\phi$ are harmonic forms on $N, \omega_{\mu}$ is a co-exact eigenform on $N$ with eigenvalue $\mu$ and $\alpha_{p-1}=(1+2(p-1)-m) / 2$. 
Then we can easily verify that

\section{COROLLARY 2.3 .}

(2.7) In case $m=2 k-1$, we have

$$
\mathscr{T}^{p}(X) \cong \begin{cases}H^{p}(M) & : p<k, \\ i^{*}\left(H^{p}(M, N)\right) \subset H^{p}(M) & : p=k, \\ H^{p}(M, N) & : p>k .\end{cases}
$$

(2.8) In case $m=2 k$, we have

$$
\mathscr{H}^{p}(X) \cong \begin{cases}H^{p}(M) & : p \leqq k, \\ H^{p}(M, N) & : p>k .\end{cases}
$$

Moreover, in case $2 p>m+1$, every element of the kernel of $\int: \mathscr{H}^{p}(X) \rightarrow \bar{H}_{(2)}^{p}\left(K^{*}\right)$ is exact on every relatively compact open set in $X$. And we have

$$
\mathscr{H}^{p}(X) \cong \begin{cases}\overline{\mathcal{H}}_{S}^{p}(X) & : 2 p<m, \\ \overline{\mathcal{H}}_{S}^{p}(X)=\overline{\mathcal{H}}_{d}^{p}(X) & : 2 p=m+1, \\ \overline{\mathcal{H}}_{d}^{p}(X) & : 2 p>m+2 .\end{cases}
$$

Proof of Corollary 2.3. (2.7) and (2.8) are rewrittings of (2.1) and (2.2). We have $\int \omega \in \overline{d_{c} C_{2}^{p-1}\left(K^{*}\right)}$, for any element $\omega$ of the kernel of $\int: \mathscr{T}^{p}(X) \rightarrow \bar{H}_{(2)}^{p}\left(K^{*}\right)$, which implies that $\int_{K \cup L_{0} \cup \cdots \cup L_{n}} \omega \in d_{c} C_{2}^{p-1}\left(K \cup L_{0} \cup \ldots \cup L_{n}\right)$ for any $n \in N$. Then $\omega_{1 M \cup C_{0}, \eta n(N)}$ is exact. (2.9) when $2 p>m+2$ can be proved as follows. Let $\varphi_{n}$ be a smooth function on $X$ which is equal to 1 on $C_{\eta n, 1}(N) \cup M$, identically 0 on $C_{0, \eta n+2}(N)$, moreover, depends only on $r$ on $C_{\eta^{n+2, \eta n}}(N)$ and $\left|\partial \varphi_{n} / \partial r\right| \leqq 1 / \eta^{n}(1-\eta)$. Then, for $\omega \in \mathscr{H}^{p}(X), \lim _{n \rightarrow \infty} \varphi_{n} \omega=\omega$ in the $L^{2}$-sense and

$$
\begin{aligned}
\lim _{n \rightarrow \infty}\left\|d\left(\varphi_{n} \omega\right)\right\|_{0} & =\lim _{n \rightarrow \infty}\left\|d\left(\varphi_{n} \omega\right)\right\|_{0 ; \eta n+2, \eta^{n}} \\
& =\lim _{n \rightarrow \infty}\left\|\frac{\partial \varphi_{n}}{\partial r} d r \wedge \omega\right\|_{0 ; \eta n+2, \eta^{n}} \\
& \leqq K \lim _{n \rightarrow \infty}\left\{\int_{\eta^{n+2}}^{\eta_{n}}\left(\frac{1}{\eta^{n}(1-\eta)}\right)^{2} r^{m-2 p+2 \alpha_{p-1}+2} \sqrt{\alpha_{p-1}^{2}+\mu} d r\right\}^{1 / 2} \\
& =K^{\prime} \lim \frac{\eta^{2 n} \sqrt{\alpha_{p-1}^{2}+\mu}-\eta^{2(n+2)} \sqrt{\alpha_{p-1}^{2}+\mu}}{\eta^{2 n}(1-\eta)^{2}}=0,
\end{aligned}
$$

which mean that $\omega \in \overline{\mathscr{H}}_{d}^{p}(X)$. (2.9) when $2 p<m$ can be easily proved by applying 
the *-operator to (2.9) when $2 p>m+2$. (2.9) when $2 p=m+1$ will be referred after the proof of (2.1).

\section{$\S 3$. The harmonic forms on $C(N)$; the proof of Theorem 2.2}

We will examine the harmonic forms on $C(N)$; see [4], [3.9] of which seems to be incorrect. The proofs are so long that we will assign them to Appendix. Here $\tilde{d}, \tilde{\delta}$ and $\tilde{\Delta}$ denote the intrinsic operations on $N$.

Proposition 3.1. The harmonic p-form $\theta(\Delta \theta=0)$ on $C(N)$ can be written as a convergent sum of forms of the following four types;

$$
\begin{array}{ll}
\phi^{ \pm}=r^{a^{ \pm}(p)} \phi & : \nu(p) \neq 0, \\
\phi^{ \pm}=\phi, \log r \cdot \phi & : \nu(p)=0,
\end{array}
$$

where $\phi$ is a co-closed eigenform of $\tilde{\Delta}$ with eigenvalue $\mu$,

$$
\begin{aligned}
\omega^{ \pm} & =r^{a^{ \pm}(p-1)} \tilde{d} \omega+a^{ \pm}(p-1) r^{a^{ \pm}(p-1)-1} d r \wedge \omega=d\left(r^{a^{ \pm}(p-1)} \omega\right) \\
& =\frac{a^{\mp}(m-p)}{\mu} \delta\left(\gamma^{-a^{\mp}(m-p)+1} d r \wedge \tilde{\delta} \omega\right),
\end{aligned}
$$

where $\omega$ is a co-exact eigenform of $\tilde{\Delta}$ with ergenvalue $\mu$,

$$
\begin{array}{cc}
\rho^{ \pm}=a^{ \pm}(p-1) \cdot r^{a^{ \pm}(p-1)+2} \rho-r^{a^{ \pm}(p-1)+1} d r \wedge \tilde{\delta} \rho & : \nu(p-1) \neq 1, \\
\rho^{+}=a^{+}(p-1) r^{a^{+}(p-1)+2} \rho-r^{a^{+}(p-1)+1} d r \wedge \tilde{\delta} \rho & : \nu(p-1)=1, \\
\rho^{-}=-\frac{2 \mu}{\left(a^{-}(p-1)+2\right)^{2}+\mu} \frac{1}{a^{-(p-1)+2} \log r \cdot \rho+r^{a-(p-1)+1} \log r d r \wedge \tilde{\delta} \rho} \\
: \nu(p-1)=1,
\end{array}
$$

where $\rho$ is an exact eigenform of $\tilde{\Delta}$ with eigenvalue $\mu$,

$$
\begin{array}{ll}
\psi^{ \pm}=r^{a^{ \pm}(p-2)+1} d r \wedge \phi & : \nu(p-2) \neq 0, \\
\phi^{ \pm}=r \cdot d r \wedge \phi, r \log r \cdot d r \wedge \phi: \nu(p-2)=0,
\end{array}
$$

where $\phi$ is a closed eigenform of $\tilde{\Delta}$ with eigenvalue $\mu$,

where $\alpha_{p}=\frac{1+2 p-m}{2}, \nu(p)=\sqrt{\alpha_{p}^{2}+\mu}$ and $a^{ \pm}(p)=\alpha_{p} \pm \nu(p)$.

Then, we can easily verify that

COROLLARY 3.2. The harmonic form $\theta(d \theta=\delta \theta=0)$ on $C(N)$ can be written as a convergent sum of forms of types; $\phi^{+}(\mu=0$ and $2 p \leqq m-1), \phi^{-}(\mu=0$ and $2 p \geqq m), \omega^{+}, \omega^{-}, \phi^{+}(\mu=0$ and $2 p \geqq m+3)$ and $\psi^{-}(\mu=0$ and $2 p \leqq m+2)$. 
COROllary 3.3. The harmonic form $\theta\left(\theta \in \Lambda^{* ; 0}\left(C_{0,1}(N)\right), d \theta=\delta \theta=0\right)$ on $C(N)$ can be written as a convergent sum of forms of types; $\phi^{+}(\mu=0$ and $2 p \leqq$ $m-1), \phi^{-}(\mu=0$ and $2 p=m), \omega^{+}, \phi^{+}(\mu=0$ and $2 p \geqq m+3)$ and $\phi^{-}(\mu=0$ and $2 p=m+2)$.

Then, Theorem 2.2 is the rewritting of Corollary 3.3.

\section{$\S 4$. The proof of Theorem 2.1}

We begin with the examination of the basic properties of the Whitney map consisting of the barycentric coordinate functions.

LEMMA 4.1. For every $p$ such that $2 p \leqq m+1$, the Whitney map $W$ induces a bounded operator

$$
W: C_{2}^{p}\left(K^{*}\right) \longrightarrow \Lambda^{p ; 0} .
$$

Proof. Let $f=\sum f_{\sigma} \cdot \sigma \in C_{2}^{p}\left(K^{*}\right)$.

$$
\begin{aligned}
W f & =\sum f_{\sigma} \cdot W \sigma \\
& =\sum_{\sigma \in K \cup L_{0} \backslash\{\eta\} \times L} f_{\sigma} \cdot W \sigma+\sum_{j=1}^{l} \sum_{n=0}^{\infty} f_{\pi\left(\tau_{\jmath}\right)} \cdot W \pi_{n}\left(\tau_{\jmath}\right) \\
& =\sum_{\sigma \in K \cup L_{0} \backslash\{\eta\} \times L} f_{\sigma} \cdot W \sigma+\sum_{j=1}^{l} \sum_{n=0}^{\infty} f_{\pi\left(\tau_{j}\right)} \cdot\left(\pi_{n}^{-1}\right) * W \tau_{\jmath},
\end{aligned}
$$

where $\tau_{1}, \cdots, \tau_{l}$ are all $p$-simplexes of $L_{1} \backslash\left\{\eta^{2}\right\} \times L$. Then, (1.10) implies

$$
\begin{aligned}
\left\|\sum_{n=0}^{\infty} f_{\pi_{n}\left(\tau_{j}\right)} \cdot\left(\pi_{n}^{-1}\right)^{*} W \tau_{j}\right\|_{0}^{2} & \leqq \sum_{n=0}^{\infty} f_{\pi_{n}\left(\tau_{j}\right)}^{2}\left\|\left(\pi_{n}^{-1}\right)^{*} W \tau_{\jmath}\right\|_{0}^{2} \\
& =\sum_{n=0}^{\infty} f_{\pi_{n}\left(\tau_{j}\right)}^{2} \cdot \eta^{n(m-2 p+1)} \cdot\left\|W \tau_{j}\right\|_{0}^{2} .
\end{aligned}
$$

Since $m-2 p+1 \geqq 0$, summing up over $j=1,2, \cdots, l$, we obtain the estimate

$$
\|W f\|_{0}^{2} \leqq C \cdot \max _{\substack{\sigma \in K \cup L^{\prime}(\{\eta) \times L \\ j=1,2, \cdots, l}}\left\{\|W \sigma\|_{0}^{2},\left\|W \tau_{j}\right\|_{0}^{2}\right\} \cdot\|f\|^{2},
$$

where $C>0$ is independent of $f$.

Besides, exchanging $K^{*}$ for $S d^{k}\left(K^{*}\right)$, where $k$ is an arbitrary positive integer, we can samely prove that $W$ induces a bounded operator

$$
W: C_{2}^{p}\left(S d^{k}\left(K^{*}\right)\right) \longrightarrow \Lambda^{p ; 0}
$$

for each $p$ such that $2 p \leqq m+1$. Moreover the norms $\|W\|$ are bounded independently of $k$.

Lemma 4.2. Set $l>(m+1) / 4+1 / 2$. Then there exists $C>0$ such that, for every $\omega \in \Lambda^{p ; l}$ and $k \in N$, we have 


$$
\left\|\omega-W_{S d k\left(K^{*}\right)} \int_{S d k\left(K^{*}\right)} \omega\right\|_{0} \leqq C \cdot h(k)^{(m+3) / 2}\|\omega\|_{l},
$$

where $h(k)$ is the mesh of $S d^{k}(K)([11])$.

Proof. For every $(m+1)$-simplex $\sigma$ of $K^{*}$, we will take a coordinate system $\left(U_{\sigma},\left(x_{\sigma}^{1}, \cdots, x_{\sigma}^{m+1}\right)\right)$ such that $U_{\sigma} \supset|\sigma|$ and $\bar{U}_{\sigma}$ is compact. Moreover we assume that, for every $(m+1)$-simplex $\sigma$ of $C_{0,1}(L)$ and every $n \in N$, the following conditions are satisfied.

(i) $\pi_{n}\left(U_{\sigma}\right)=U_{\pi_{n}(\sigma)}$.

(ii) The coordinate functions of $U_{\pi_{n}(\sigma)}$ are

$$
x_{\pi_{n}(\sigma)}^{1}=x_{\sigma}^{1} \circ \pi_{-n}, x_{\pi_{n}(\sigma)}^{2}=x_{\sigma}^{2} \circ \pi_{-n}, \cdots, x_{\pi_{n}(\sigma)}^{m+1}=x_{\sigma}^{m+1} \circ \pi_{-n} .
$$

Since $S d^{k}\left(K^{*}\right)$ is a subdivision of $K^{*}$, for every $(m+1)$-simplex $\tau$ of $S d^{k}\left(K^{*}\right)$, we have an unique $(m+1)$-simplex $\sigma$ of $K^{*}$ such that $|\tau| \subset|\sigma|$.

Let $\sigma$ be an $(m+1)$-simplex of $S d^{k}\left(K \cup L_{0} \cup L_{1}\right)$. Then there exists a constant $C>0$, independent of $\sigma, \omega$ and $k$, such that, for every $p$-form $\omega$ and every $k \in N$.

$$
\sup _{x \in \mid \sigma_{1}}\left|\omega(x)-\left(W_{S d k\left(K^{*}\right)} \int_{S d^{k}\left(K^{*}\right)} \omega\right)(x)\right| \leqq C \operatorname{diam} \sigma \sup _{x \in|\sigma|}\left|\frac{\partial \omega}{\partial r}(x)\right|
$$

because of the compactness of $\overline{C_{\eta^{3}, 1}(N)} \cup M,[7]$ and [9].

Let $\sigma_{1}, \cdots, \sigma_{l}$ be all $(m+1)$-simplexes of $L_{1} \backslash\left\{\eta^{2}\right\} \times L$ and $U_{1}, \cdots, U_{l}$ all their coordinate neighborhoods as above. Let $(r, x) \in \pi_{n}\left(\sigma_{\jmath}\right)$.

$$
\begin{aligned}
& \left|\omega(r, x)-\left(W_{S d^{k}\left(K^{*}\right)} \int_{S d^{k}\left(K^{*}\right)} \omega\right)(r, x)\right|^{2} \\
& =\eta^{-2 p n}\left|\left(\pi_{n}^{*} \omega\right)\left(\eta^{-n} r, x\right)-\left(\pi_{n}^{*} W_{S d k\left(K^{*}\right)} \int_{S d^{k}\left(K^{*}\right)} \omega\right)\left(\eta^{-n} r, x\right)\right|^{2} \quad \text { (by (1.8)) } \\
& =\eta^{-2 p n}\left|\left(\pi_{n}^{*} \omega\right)\left(\eta^{-n} r, x\right)-\left(W_{S d k\left(K^{*}\right)} \int_{S d^{k}\left(K^{*}\right)} \pi_{n}^{*} \omega\right)\left(\eta^{-n} r, x\right)\right|^{2} \\
& \leqq \eta^{-2 p n} C^{2}\left(\operatorname{diam} \sigma_{\jmath}\right)^{2} \sup _{y \in\left|\sigma_{\jmath}\right|}\left|\frac{\partial \pi_{n}^{*} \omega}{\partial y}(y)\right|^{2} \\
& \leqq \eta^{-2 p n} C^{2}\left(\operatorname{diam} \sigma_{\jmath}\right)^{2} C_{1}\left\|\pi_{n}^{*} \omega\right\|_{l ; U_{\jmath}}^{2} \quad \text { (Sobolev lemma) } \\
& \leqq \eta^{-n(m+1)} C^{2} C_{1}^{2} h(k)^{2}\|\omega\|_{l ; \pi_{n}\left(U_{\jmath}\right)}^{2} \quad \text { (by (1.9) and (1.10)), }
\end{aligned}
$$

where $h(k)$ is the mesh of $S d^{k}\left(K \cup L_{0} \cup L_{1}\right)([11])$ and $C_{1}>0$ is independent of $j$ and $\omega$.

Therefore, we have

$$
\int_{\pi_{n}\left(\sigma_{j}\right)}\left|\omega(r, x)-\left(W_{S d k\left(K^{*}\right)} \int_{S d^{k}\left(K^{*}\right)} \omega\right)(r, x)\right|^{2} d V
$$




$$
\begin{aligned}
& \leqq C^{2} C_{1}^{2} \eta^{-n(m+1)} h(k)^{2}\|\omega\|_{l ; \pi_{n}\left(U_{j}\right)}^{2}\left(\eta^{n} \operatorname{diam} \sigma_{\jmath}\right)^{m+1} \\
& \leqq C^{2} C_{1}^{2} h(k)^{m+3}\|\omega\|_{l ; \pi_{n}\left(U_{\jmath}\right)}^{2}
\end{aligned}
$$

where $d V$ is the volume element of $X$.

On the other hand, for every $(m+1)$-simplex $\sigma$ of $K \cup L_{0}$, we have

$$
\int_{\sigma}\left|\omega(r, x)-\left(W_{S d k\left(K^{*}\right)} \int_{S d^{k}\left(K^{*}\right)} \omega\right)(r, x)\right|^{2} d V \leqq C_{2} h(k)^{m+3}\|\omega\|_{l ; U_{\sigma}}^{2} .
$$

Hence,

$$
\begin{aligned}
& \left\|\omega-W_{S d k\left(K^{*}\right)} \int_{S d^{k}\left(K^{*}\right)} \omega\right\|_{0}^{2} \\
& \leqq \sum_{\sigma \in K \cup L_{0} \backslash(\eta) \times L} \int_{\sigma}\left|\omega(r, x)-\left(W_{S d k\left(K^{*}\right)} \int_{S d^{k}\left(K^{*}\right)} \omega\right)(r, x)\right|^{2} d V \\
& \quad+\sum_{j=1}^{l} \sum_{n=0}^{\infty} \int_{\pi_{n}\left(\sigma_{j}\right)}\left|\omega(r, x)-\left(W_{S d k\left(K^{*}\right)} \int_{S d^{k}\left(K^{*}\right)} \omega\right)(r, x)\right|^{2} d V \\
& \leqq \\
& C_{3} h(k)^{m+3}\|\omega\|_{l}^{2} .
\end{aligned}
$$

Then, we have

Proposition 4.3. For every $p$ such that $2 p \geqq m+1$,

$$
\int: \mathscr{H}^{p}(X) \longrightarrow H_{(2)}^{p}\left(K^{*}\right)
$$

is injective.

Proof. Suppose $\left[\int_{K^{*}} \omega\right]=0$ for $\omega \in \mathscr{H}^{p}(X)$. Since the following diagram is commutative, for every $k \in N,\left[\int_{S d^{k}\left(K^{*}\right)} \omega\right]=0$.

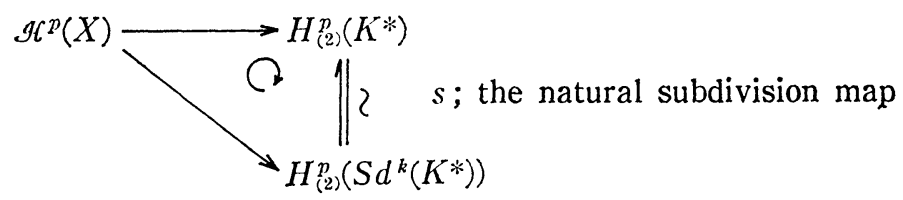

Therefore, for every $k \in N$, there exists $f=\sum f_{\sigma} \cdot \sigma \in C_{2}^{p-1}\left(K^{*}\right)$ such that

$$
\int_{S d^{k}\left(K^{*}\right)} \omega=d_{c} f
$$

Hence, if we put $\omega=\omega_{1}+d r \wedge \omega_{2}$, we have 


$$
\begin{aligned}
& \left|\left(W_{S d k\left(K^{*}\right)} \int_{S d^{k}\left(K^{*}\right)} \omega, \omega\right)_{0 ; C_{\eta},_{1}(N) \cup M}\right|=\left|\left(d W_{S d k\left(K^{*}\right)} f, \omega\right)_{0 ; C_{\eta} n_{1}}(N) \cup M\right| \\
& =\left|\int_{\left(\eta^{n}\right) \times N}\left(W_{S d k\left(K^{*}\right)} f\right)_{\tan } \wedge \tilde{*} \omega_{\text {norm }}\right| \\
& =\left|\int_{\left\{\eta n_{1 \times N}\right.} W_{\{\eta n] \times S d k(L)}\left(f_{\{i \eta n] \times S d k(L)}\right) \wedge \tilde{*} \omega_{2}\right| \\
& =\mid \int_{1 \times N} W_{1 \times S d k(L)}\left(\pi_{-n}\left(f_{1(\eta n) \times S d k(L))}\right) \wedge \eta^{n(m-2(p-1))} \tilde{*} \pi_{n}^{*} \omega_{2} \mid \text { by }(1.7)\right) \\
& \leqq C \eta^{n(m-2(p-1)+a)}\left|\int_{1 \times N} W_{1 \times S d k(L)}\left(\pi_{-n}\left(f_{\mid(\eta n) \times S d k(L)}\right)\right) \wedge \widetilde{*} \xi\right| \\
& \leqq C\left|\int_{1 \times N} \sum_{\sigma \in 1 \times S d^{k}(L)} f_{\pi_{n}(\sigma)} W_{1 \times S d k(L)} \sigma \wedge \tilde{*} \xi\right| \\
& =C \sum_{\sigma \in 1 \times S d^{k}(L)}\left|f_{\pi_{n}(\sigma)}\right| \cdot\left|\int_{1 \times N} W_{1 \times S d k(L)} \sigma \wedge \widetilde{*} \xi\right| \\
& =C \sum_{\sigma \in 1 \times S d^{k}(L)}\left|f_{\pi_{n}(\sigma)}\right|\left(\sum_{\sigma \in 1 \times S d^{k}(L)}\left|\int_{1 \times N} W_{1 \times S d k(L)} \sigma \wedge \tilde{*} \xi\right|\right),
\end{aligned}
$$

where $a$ and $\xi$ can be determined according to Corollary 3.3 and, in any case, we know that $m-2(p-1)+a \geqq 0$. And we know that $\lim _{n \rightarrow \infty} \sum_{\sigma \in 1 \times S d^{k}(L)}\left|f_{\pi_{n}(\sigma)}\right|=0$, which yields

$$
\lim _{n \rightarrow \infty}\left|\left(W_{S d^{n}\left(K^{*}\right)} \int_{S d^{k}\left(K^{*}\right)} \omega, \omega\right)_{0 ; C_{\eta},{ }_{1}(N) \cup M}\right|=0 .
$$

On the other hand, from Corollary 3.3 and Lemma 4.2, we have

$$
\lim _{n \rightarrow \infty}\|\omega\|_{0 ; C_{0}, \eta n(N)}=0
$$

and

$$
\begin{aligned}
\lim _{n \rightarrow \infty} \| & W_{S d k\left(K^{*}\right)} \int_{S d^{k}\left(K^{*}\right)} \omega \|_{0 ; C_{0}, \eta n(N)} \\
& \leqq \lim _{n \rightarrow \infty}\left\{\left\|\omega-W_{S d k\left(K^{*}\right)} \int_{S d^{k}\left(K^{*}\right)} \omega\right\|_{0: C_{0}, \eta n(N)}+\|\omega\|_{0 ; C_{0, \eta} n(N)}\right\} \\
& \leqq \lim _{n \rightarrow \infty}\left\{C \cdot h(k)^{(m+3) / 2}\|\omega\|_{0 ; C_{0}, \eta n(N)}+\|\omega\|_{0 ; C_{0, \eta} n(N)}\right\}=0 .
\end{aligned}
$$

Therefore, we have 


$$
\begin{aligned}
& \left|\left(W_{S d k\left(K^{*}\right)} \int_{S d^{k}\left(K^{*}\right)} \omega, \omega\right)_{0 ; \eta_{\eta}, 1_{1}(N) \cup M}-\left(W_{S d k\left(K^{*}\right)} \int_{S d^{k}\left(K^{*}\right)} \omega, \omega\right)_{0 ; X}\right| \\
& \quad \leqq\left|\left(W_{S d k\left(K^{*}\right)} \int_{S d^{k}\left(K^{*}\right)} \omega, \omega\right)_{0 ; C_{0, \eta}(N)}\right| \\
& \quad \leqq\left\|W_{S d^{k}\left(K^{*}\right)} \int_{S d^{k}\left(K^{*}\right)} \omega\right\|_{0 ; C_{0}, \eta_{\eta}(N)} \cdot\|\omega\|_{0 ; C_{0, \eta} n(N) n \underset{n \rightarrow \infty}{\longrightarrow} \infty} \infty .
\end{aligned}
$$

This, combined with (4.1), implies

$$
\left(W_{S d k\left(K^{*}\right)} \int_{S d^{k}\left(K^{*}\right)} \omega, \omega\right)_{0 ; X}=0 \quad \text { for every } k \in N .
$$

Therefore, Lemma 4.2 and (4.2) imply $\omega=0$.

On the other hand, from Corollary 3.3, we can easily verify that

Proposition 4.4. For each $p$ such that $2 p \geqq m+1$, the image of

$$
\int: \mathscr{H}^{p}(X) \longrightarrow H_{(2)}^{p}\left(K^{*}\right)
$$

is contained in $i_{2}\left(H^{p}(K, L)\right)$.

Proof. It suffices to prove that $j_{2}\left(\int \omega\right) \in d_{c, C_{0,1}(L)} C_{2}^{p-1}\left(C_{0,1}(L)\right)$ for any $\omega \in$ $\mathscr{H}^{p}(X)$. Since $2 p \geqq m+1$, Corollary 3.3 implies that $\omega_{1 C_{0,1}(N)}$ can be written as a convergent sum of types; $\omega^{+}, \psi^{+}(\mu=0$ and $2 p \geqq m+3)$ and $\psi^{-}(\mu=0$ and $2 p=$ $m+2)$. Hence we should prove that $\int_{C_{0,1}(L)} r^{r^{+(p-1)}} \omega$ and $\int_{C_{0,1}(L)} r^{2 p-m-1} \psi(\mu=0$ and $2 p \geqq m+2)$ belong to $C_{2}^{p-1}\left(C_{0,1}(L)\right)$, which are straight-forward.

Now we will prove (2.1) of Theorem 2.1. From Proposition 4.3 and 4.4, it suffices to prove $\operatorname{dim} \mathscr{H}^{p}(X) \geqq \operatorname{dim} H^{p}(M, N)$ for $2 p>m+1$, which is equivalent to $\operatorname{dim} \mathscr{H}^{p}(X) \geqq \operatorname{dim} H^{p}(M)$ for $2 p<m+1$. Then we will prove the latter. It is well-known that $\mathcal{H}_{\text {norm }}^{p}(M)=\left\{\theta \in \Lambda^{p}(M) \mid d \theta=\delta \theta=0\right.$ and $\theta_{\text {norm }}=0$ on $\left.\partial M=N\right\}$ is isomorphic to $H^{p}(M)$ by the inclusion ([10]). Then, let $\tilde{\theta}$ be the $p$-form which is equal to $\theta \in \mathcal{H}_{\text {norm }}^{p}(M)$ on $M$ and equal to the natural extension of $\theta_{1 \partial M}$ on $C_{0,1}(N)$. It is easily verified by using (1.5) and the assumption $2 p<m+1$ that $\tilde{\theta}$ belongs to $A^{p ; 0}(X)$. Moreover, $d \tilde{\theta}=0$ holds in the weak sense; i.e. for any $\beta \in \Lambda_{0}^{p}(X),(\tilde{\theta}, \delta \beta)_{0}=0$, which, using the Friedrichs molifier ([8]), implies $\tilde{\theta} \in \operatorname{dom} \bar{d}$ and $\bar{d} \tilde{\theta}=0$ i.e. $\tilde{\theta} \in \operatorname{ker} \bar{d}_{p}=\mathscr{T}^{p}(X) \oplus \overline{d \Lambda_{0}^{p-1}(X)}$; see Lemma 1.3. We define $\tilde{\theta}$ as the harmonic part of $\tilde{\theta}$. Then the linear map $\mathscr{H}_{\text {norm }}^{p}(M) \ni \theta \mapsto \tilde{\theta} \in \mathscr{H}^{p}(X)$ is injective. The proof is as follows. Suppose $\tilde{\theta}=0$. Then $\tilde{\theta} \in \overline{d \Lambda_{0}^{p-1}(X)}$, which implies $\theta \in \overline{d \Lambda^{p-1}(M)}$. But, for any $\alpha \in \Lambda^{p-1}(M),(d \alpha, \theta)_{0 ; M}=(\alpha, \delta \theta)_{0 ; M}+\left(\alpha_{\tan }, \theta_{\text {norm }}\right)_{0 ; \partial M}$ $=0$; i. e. $\theta$ is orthogonal to $\overline{d \Lambda^{p-1}(M)}$. Therefore $\theta=0$. Thus the proof of $\operatorname{dim} \mathscr{C}^{p}(X) \geqq \operatorname{dim} H^{p}(M)$ for $2 p<m+1$ is complete.

Note that, if $2 p=m+1$, then $\tilde{\theta}$ does not belong to $A^{p ; 0}(X)$ except the case 
$\theta_{\mid \partial M}=0$. Therefore, we cannot apply the above method to the case $2 p=m+1$.

Then, we will prove (2.2) of Theorem 2.1. In this case, both the bounded de Rham map and the bounded Whitney map can be defined.

$$
\int: \Lambda^{p ; l}(X) \longrightarrow C_{2}^{p}\left(S d^{k}\left(K^{*}\right)\right) \quad \text { for } l>(m+1) / 4+1 / 2 \text { and } k \in N .
$$

$$
W: C_{2}^{p}\left(S d^{k}\left(K^{*}\right)\right) \longrightarrow \Lambda^{p ; 0}(X) \quad \text { for } k \in N .
$$

First, we will prove that $\int: \mathscr{T}^{p}(X) \rightarrow \imath_{2}\left(\imath_{1}\left(H^{p}(K, L)\right)\right)$ is injective. Suppose $i_{2}\left(i_{1}\left(H^{p}(K, L)\right)\right)=\bar{H}_{(2)}^{p}\left(K^{*}\right) \ni\left[\int_{K^{*}} \omega\right]=0$ for $\omega \in \mathscr{H}^{p}(X)$. Since the following diagram is commutative for every $k \in N$, we have $\left[\int_{S d^{k}\left(K^{*}\right)} \omega\right]=0$.

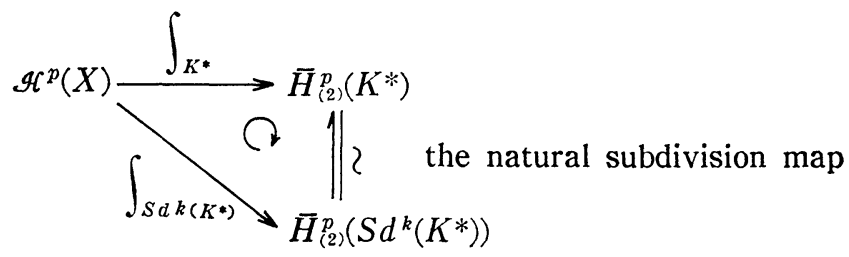

For every $\varepsilon_{1}>0$, from Lemma 4.2 , we can choose $k \in N$ such that

$$
\left\|\omega-W_{S d^{k}\left(K^{*}\right)} \int_{S d^{k}\left(K^{*}\right)} \omega\right\|_{0}<\varepsilon_{1} .
$$

Now, since $\int_{S d^{k}\left(K^{*}\right)} \omega \in \overline{d_{c}\left(C_{2}^{p-1}\left(S d^{k}\left(K^{*}\right)\right)\right)}$ and $d_{c}$ is bounded, we can find, given arbitrary $\varepsilon_{2}>0$, a finite cochain $f \in C_{2}^{p-1}\left(S d^{k}\left(K^{*}\right)\right)$ such that

$$
\left\|\int_{S d^{k}\left(K^{*}\right)} \omega-d_{c} f\right\|<\varepsilon \text {. }
$$

Therefore, we have

$$
\begin{aligned}
& \left\|\omega-d W_{S d k\left(K^{*}\right)} f\right\|_{0} \\
& \quad \leqq\left\|\omega-W_{S d k\left(K^{*}\right)} \int_{S d^{k}\left(K^{*}\right)} \omega\right\|_{0}+\left\|W_{S d k\left(K^{*}\right)}\right\| \cdot\left\|\int_{S d^{k}\left(K^{*}\right)} \omega-d_{c} f\right\| \\
& \quad \leqq \varepsilon_{1}+\left\|W_{S d k\left(K^{*}\right)}\right\| \cdot \varepsilon_{2} .
\end{aligned}
$$

Since $\left\|W_{S d^{k\left(K^{*}\right)}}\right\|$ is bounded independently of $k$ and $d W_{S d^{k\left(K^{*}\right)}} f$ belongs to $\overline{d \Lambda_{0}^{p-1}(X)}$, this shows that $\omega \in \overline{d \Lambda_{0}^{p-1}(X)}$, i. e. $\omega=0$.

Next, we will prove that $\int: \mathscr{H}^{p}(X) \rightarrow i_{2}\left(\imath_{1}\left(H^{p}(K, L)\right)\right)$ is surjective. Let $f_{1}$, $\cdots, f_{n}$ be finite cocycles consisting of $p$-simplexes of $K \backslash L$ whose images in $\bar{H}_{(2)}^{p}\left(K^{*}\right)=i_{2}\left(i_{1}\left(H^{p}(K, L)\right)\right)$ form a basis of $\bar{H}_{(2)}^{p}\left(K^{*}\right)$. Let $f$ be a non-zero linear 
combination of these elements. Then, for the smooth Whitney map $W, \int W f=f$. Therefore, when we decompose $W f$ into $W f=\omega+\nu$, where $\omega \in \mathscr{H}^{p}(X)$ and $\nu \in$ $\overline{d \Lambda_{0}^{p-1}(X)}$, it suffices to prove that $\int \nu$ belongs to $\overline{d_{c} C_{2}^{p-1}\left(K^{*}\right)}$. Since $\nu=W f-\omega$ $\in \operatorname{ker}\left(d: \Lambda^{p ; l} \rightarrow \Lambda^{p ; l-1}\right)$ for arbitrary $l, \int \nu$ belongs to ker $d_{c, p}$. We can decompose $\int \nu$ as follows;

$$
\int \nu=h+d_{c, p-1} a+g
$$

where $h \in \operatorname{ker}\left(d_{c, p}=d_{c, p,(K, L)}: C^{p}(K, L) \rightarrow C^{p+1}(K, L)\right), \quad a \in C^{p-1}(L)$ and $g \in$ $\left.\overline{d_{c} C_{2}^{p-1}\left(K^{*} \backslash(K \backslash L), L\right.}\right)$ because of (1.3) and (1.4). Therefore $\int_{K} \nu=h+d_{c, p-1, K} a$ belongs to $\operatorname{ker}\left(d_{c, p, K}: C^{p}(K) \rightarrow C^{p+1}(K)\right)$. Then it suffices to prove $h \in$ $d_{c, p-1} C^{p-1}(K, L)$. Suppose $h \notin d_{c, p-1} C^{p-1}(K, L)$. Then, $\int_{K} \nu \notin d_{c, p-1, K} C^{p-1}(K)$ and we can find the harmonic form $\varphi$ on $M$ which satisfies the absolute boundary condition ;

$$
\varphi_{\mathrm{norm}}=(d \varphi)_{\mathrm{norm}}=0 \quad \text { on } \partial M=N
$$

such that $\int_{K} \varphi-\int_{K} \nu \in d_{c, p-1}{ }_{K} C^{p-1}(K)$, because the space of such harmonic forms on $M$ is isomorphic to $H^{p}(K)$ by the de Rham map $\int_{K}([10])$. On the other hand, by using non-smooth Whitney maps, $\varphi$ and $\nu_{\mid M}$ can be approximated by $W_{S d k(K)} \int_{S d^{k}(K)} \varphi$ and $W_{S d^{k(K)}} \int_{S^{k}{ }^{k}(K)} \nu_{\mid M}$ in the $L^{2}$-sense when $k \rightarrow \infty$, respectively (Lemma 4.2, [7] and [9]). Therefore, $\varphi=\nu_{\mid M}+\left(\varphi-\nu_{\mid M}\right)$ can be approximated by elements of $d \Lambda^{p-1}(M)$ in the $L^{2}$-sense. But $\varphi$ is orthogonal to $d \Lambda^{p-1}(M)$, which is a contradiction.

Thus the proof of (2.2) of Theorem 2.1 is complete.

Then, we refer to $(2.9)$ when $2 p=m+1$. Since $W f \in \operatorname{ker} \tilde{d}_{p}=\overline{\mathscr{H}}_{d}^{p}(X) \oplus \overline{d \wedge_{0}^{p-1}(X)}$ for $f$ in the proof of the surjectiveness of $\int: \mathscr{H}^{p}(X) \rightarrow \imath_{2}\left(\imath_{1}\left(H^{p}(K, L)\right)\right)$, the harmonic part $\omega$ of $W f$ belongs to $\overline{\mathscr{H}}_{d}^{p}(X)$. Therefore $\mathscr{T}^{p}(X)=\overline{\mathscr{H}}_{d}^{p}(X)$. Moreover, $\mathscr{H}^{p}(X)=\widehat{\mathscr{H}}_{\delta}^{p}(X)$ is easily verified by using the action of the $*$-operator.

Finally, we refer to (2.3) of Theorem 2.1. If, for $\omega \in \mathcal{H}^{p}(X), \int \omega$ belongs to $C_{2}^{p}\left(K^{*}\right)$, then $\omega_{\mid M}$ is exact because of (1.4). Let $\mathscr{T}_{e}^{p}(X)$ be the subspace of $\mathscr{H}^{p}(X)$ consisting of forms whose restrictions to $N$ are exact. Then, the restriction of any element of $\mathcal{T}_{e}^{p}(X)$ to $C_{0,1}(N)$ can be written as a convergent sum of forms of type $d\left(r^{a+(p-1)} \omega_{\mu}\right)$; see (2.6) and Corollary 3.3. Therefore, for $\omega \in \mathscr{T}^{p}(X)$, 
$\int \omega$ belongs to $C_{2}^{p}\left(K^{*}\right)$ if and only if $\omega \in \mathcal{F}_{e}^{p}(X)$ (cf. the proof of Proposition 4.4). And $\int: \mathscr{T}_{e}^{p}(X) \rightarrow \bar{H}_{(2)}^{p}\left(K^{*}\right)$ is injective. It is samely proved as the proof of the injectiveness of $\int: \mathscr{K}^{p}(X) \rightarrow \imath_{2}\left(\imath_{1}\left(H^{p}(K, L)\right)\right)$ when $2 p=m+1$. Therefore, we have $\operatorname{dim} \mathcal{H}_{e}^{p}(X) \leqq \operatorname{dim} i^{*}\left(H^{p}(M, N)\right)$, i. e. $\mathcal{H}_{e}^{p}(X)$ is, in general, not equal to $\mathscr{H}^{p}(X)$.

\section{$\S 5$. Appendix}

We will prove Proposition 3.1, Corollary 3.2 and 3.3.

Let $N^{m}$ be an $m$-dimensional oriented closed Riemannian manifold. Let $C(N)$ be the space $(0, \infty) \times N$ with metric $d r \otimes d r+r^{2} g$, where $g$ is the metric on $N$, Let $\bar{d}, \tilde{\delta}, \tilde{*}$ and $\tilde{\Delta}$ be the exterior derivative, its formal adjoint, the $*$-operator and the Laplacian on $N$. Let $d, \delta$, and $\Delta$ be those on $C(N)$.

LEMMA 5.1. Let $\theta(r, x)=g(r) \phi(x)+f(r) d r \wedge \omega(x)$ be an element of $\wedge^{p}(C(N))$, where $g, f$ are smooth functions of $r \in(0, \infty)$ and $\phi, \omega$ are smooth $p$ and $(p-1)$ forms on $N$, respectively. Then, we have

$$
\begin{aligned}
& * \theta=(-1)^{p} r^{m-2 p} g d r \wedge \tilde{*} \phi+r^{m-2(p-1)} f \tilde{*} \omega, \\
& d \theta=g \tilde{d} \phi+g^{\prime} d r \wedge \phi-f d r \wedge \tilde{d} \omega, \\
& \delta \theta=r^{-2} g \tilde{\delta} \phi-(m-2(p-1)) r^{-1} f \omega-f^{\prime} \omega-r^{-2} f d r \wedge \tilde{\delta} \omega
\end{aligned}
$$

and

$$
\begin{aligned}
\Delta \theta= & \left\{-g^{\prime \prime}-(m-2 p) r^{-1} g^{\prime}\right\} \phi+r^{-2} g \tilde{\Delta} \phi-2 r^{-3} g d r \wedge \tilde{\delta} \phi \\
& +\left\{-f^{\prime \prime}-(m-2(p-1)) r^{-1} f^{\prime}+(m-2(p-1)) r^{-2} f\right\} d r \wedge \omega \\
& +r^{-2} f d r \wedge \tilde{\Delta} \omega-2 r^{-1} f \tilde{d} \omega .
\end{aligned}
$$

Proof. (5.1) and (5.2) are obvious. (5.3) is easily verified by noticing $\delta=$ $(-1)^{(p-1)(m+1)+1} * d *$. Then we have

$$
\begin{aligned}
d \delta \theta= & -2 r^{-3} g d r \wedge \tilde{\delta} \phi+r^{-2} g^{\prime} d r \wedge \tilde{\delta} \phi+r^{-2} g \tilde{d} \tilde{\delta} \phi \\
& +(m-2(p-1)) r^{-2} f d r \wedge \omega-(m-2(p-1)) r^{-1} f^{\prime} d r \wedge \omega-(m-2(p-1)) r^{-1} f \tilde{d} \omega \\
& -f^{\prime \prime} d r \wedge \omega-f^{\prime} \tilde{d} \omega+r^{-2} f d r \wedge \tilde{d} \tilde{\delta} \omega
\end{aligned}
$$

and

$$
\begin{aligned}
\delta d \theta= & r^{-2} g \tilde{\delta} \tilde{d} \phi-(m-2 p) r^{-1} g^{\prime} \phi-g^{\prime \prime} \phi-r^{-2} g^{\prime} d r \wedge \tilde{\delta} \phi \\
& +(m-2 p) r^{-1} f \tilde{d} \omega+f^{\prime} \tilde{d} \omega+r^{-2} f d r \wedge \tilde{\delta} \tilde{d} \omega,
\end{aligned}
$$

which imply (5.4).

Now, we will investigate the harmonic forms $\theta(\Delta \theta=0)$ on $C(N)$. Let 
$\left\{\omega_{j(h, p)}, \omega_{j(c e, p)}, \omega_{j(e, p)}\right\}_{j(h, p), j(c e, p), \jmath(e, p)}$ be the orthonormal base of $\Lambda^{p}(N)$ consisting of harmonic forms, co-exact eigenforms and exact eigenforms of $\tilde{\Delta}$. The

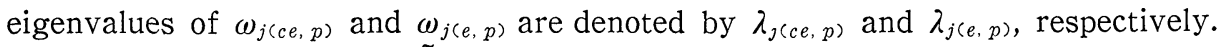
Note that $\left.\left\{\omega_{j(h, p)}, \omega_{j(c e, p)}, \tilde{d} \omega_{j(c e, p-1}\right)\right\}_{j(h, p), j(c e, p), j(c e, p-1)}$ is also the orthogonal basis of $\Lambda^{p}(N)$ and $\tilde{d} \omega_{j(c e, p-1)}$ has the eigenvalue $\lambda_{j(c e, p-1)}$. Then, we will expand $\theta \in \Lambda^{p}(C(N))$ as follows ;

$$
\begin{aligned}
\theta(r, x)= & \sum_{\jmath(h, p)} g_{\jmath(h, p)}(r) \omega_{\jmath(h, p)}(x)+\sum_{\jmath(c e, p)} g_{j(c e, p)}(r) \omega_{j(c e, p)}(x) \\
& +\sum_{\jmath(c e, p-1)} g_{j(c e, p-1)}(r) \tilde{d} \omega_{\jmath(c e, p-1)}(x) \\
& +\sum_{\jmath(h, p-1)} f_{j(h, p-1)}(r) d r \wedge \omega_{j(h, p-1)}(x) \\
& +\sum_{j(c e, p-1)} f_{j(c e, p-1)}(r) d r \wedge \omega_{j(c e, p-1)}(x) \\
& +\sum_{j(e, p-1)} f_{j(e, p-1)}(r) d r \wedge \omega_{j(e, p-1)}(x)
\end{aligned}
$$

whose derivatives converge on every relatively compact open subset of $C(N)$. Then, from (5.4), we have

$$
\begin{aligned}
\Delta \theta= & \sum_{\jmath(h, p)}\left\{-g_{\jmath}^{\prime \prime(h, p)}-(m-2 p) r^{-1} g_{\jmath}^{\prime}(h, p)\right\} \omega_{j(h, p)} \\
& +\sum_{\jmath(c e, p)}\left\{-g_{\jmath(c e, p)}^{\prime \prime}-(m-2 p) r^{-1} g_{j(c e, p)}^{\prime}+\lambda_{j(c e, p)} r^{-2} g_{j(c e, p)}\right\} \omega_{j(c e, p)} \\
& +\sum_{\jmath(c e, p-1)}\left\{-g_{\jmath(c e, p-1)}^{\prime \prime}-(m-2 p) r^{-1} g_{\jmath(c e, p-1)}^{\prime}\right. \\
& \left.\quad+\lambda_{\jmath(c e, p-1)} r^{-2} g_{\jmath \jmath(c e, p-1)}-2 r^{-1} f_{j(c e, p-1)}\right\} \tilde{d} \omega_{j(c e, p-1)} \\
& +\sum_{\jmath(h, p-1)}\left\{-f_{\jmath}^{\prime \prime}(h, p-1)-(m-2(p-1)) r^{-1} f_{j(h, p-1)}^{\prime}\right. \\
& \left.\quad+(m-2(p-1)) r^{-2} f_{j(h, p-1)}\right\} d r \wedge \omega_{j(h, p-1)} \\
& +\sum_{\jmath(c e, p-1)}\left\{-2 \lambda_{j(c e, p-1)} r^{-3} g_{j(c e, p-1)}-f_{\jmath(c e, p-1)}^{\prime \prime}\right. \\
& \quad-(m-2(p-1)) r^{-1} f_{j(c e, p-1)}^{\prime}+(m-2(p-1)) r^{-2} f_{j(c e, p-1)} \\
& \left.\quad+\lambda_{j(c e, p-1)} r^{-2} f_{j(c e, p-1)}\right\} d r \wedge \omega_{j(c e, p-1)} \\
& +\sum_{\jmath(e, p-1)}\left\{-f_{\jmath(c, p-1)}^{\prime \prime}-(m-2(p-1)) r^{-1} f_{j(e, p-1)}^{\prime}+(m-2(p-1)) r^{-2} f_{\jmath(e, p-1}\right) \\
& \left.\quad+\lambda_{j(e, p-1)} r^{-2} f_{j(e, p-1)}\right\} d r \wedge \omega_{\jmath(e, p-1)}
\end{aligned}
$$

Therefore, $\Delta \theta=0$ if and only if $(5.6)=0,(5.7)=0,(5.8)=0,(5.9)=0,(5.10)=0$ and $(5.11)=0$, which are Euler's differential equations. And the solutions of $(5.6)=0$ and $(5.7)=0$ are (3.1). The solutions of $(5.9)=0$ and $(5.11)=0$ are (3.4). Finally, the solutions of $(5.8)=0$ and $(5.10)=0$ are (3.2) and (3.3). Now we prove them 
actually.

To solve $(5.6)=0$ and $(5.7)=0$, it suffices to solve

$$
-g^{\prime \prime}-(m-2 p) r^{-1} g^{\prime}+\mu r^{-2} g=0 \quad \text { for } \mu \geqq 0 .
$$

Set $g=r^{a}$. Then (5.12) holds if and only if

$$
-a(a-1)-(m-2 p) a+\mu=0, \quad \text { i. e. } a^{2}-(1+2 p-m) a-\mu=0 .
$$

Consequently,

$$
a=\frac{1+2 p-m \pm \sqrt{(1+2 p-\bar{m})^{2}+4 \mu}}{2}=a^{ \pm}(p) .
$$

Therefore, when $\nu(p) \neq 0,(5.6)=0$ and $(5.7)=0$ if and only if

$$
\sum_{\jmath(h, p)} g_{\jmath(h, p)} \omega_{j(h, p)}+\sum_{j(c e, p)} g_{j(c e, p)} \omega_{j(c e, p)}
$$

can be written as a convergent sum of forms of types; $\phi^{ \pm}$; see (3.1). When $\nu(p)=0$, it is obvious that $(5.6)=0$ and $(5.7)=0$ if and only if (5.13) can be written as a convergent sum of forms of types; $\phi$ and $\log r \cdot \phi$; see (3.1).

To solve $(5.9)=0$ and $(5.11)=0$, it suffices to solve

$$
-f^{\prime \prime}-(m-2(p-1)) r^{-2} f^{\prime}+(m-2(p-1)) r^{-1} f+\mu r^{-2} f=0 \quad \text { for } \mu \geqq 0 .
$$

Set $f=r^{a+1}$. Then (5.14) holds if and only if

$$
\begin{gathered}
-(a+1) a-(m-2(p-1))(a+1)+(m-2(p-1)+\mu)=0, \\
\text { i. e. } a^{2}-(1+2(p-2)-m) a-\mu=0 .
\end{gathered}
$$

Cosequently,

$$
a=\frac{1+2(p-2)-m \pm \sqrt{(1+2(p-2)-m)^{2}+4 \mu}}{2}=a^{ \pm}(p-2) .
$$

Therefore, when $\nu(p-2) \neq 0,(5.9)=0$ and $(5.11)=0$ if and only if

$$
\sum_{\jmath(h, p-1)} f_{j(h, p-1)} d r \wedge \omega_{j(h, p-1)}+\sum_{j(e, p-1)} f_{\jmath(e, p-1)} d r \wedge \omega_{j(e, p-1)}
$$

can be written as a convergent sum of forms of types; $\phi^{ \pm}$; see (3.4). When $\nu(p-2)=0$, it is trivial that $(5.9)=0$ and $(5.11)=0$ if and only if $(5.15)$ can be written as a convergent sum of forms of types; $r d r \wedge \phi$ and $r \log r d r \wedge \phi$; see (3.4).

Finally, to solve $(5.8)=0$ and $(5.10)=0$, it suffices to solve the following simultaneous differential equations ;

$$
-g^{\prime \prime}-(m-2 p) r^{-1} g^{\prime}+\mu r^{-2} g-2 r^{-1} f=0
$$

and

$$
-2 \mu r^{-3} g-f^{\prime \prime}-(m-2(p-1)) r^{-1} f^{\prime}+(m-2(p-1)) r^{-2} f+\mu r^{-2} f=0 \text { for } \mu>0 .
$$


Set $g=r^{a}$ and $f=c r^{a-1}$. Then (5.16) and (5.17) hold if and only if

$$
-a(a-1)-(m-2 p) a+\mu-2 c=0
$$

and

$$
-2 \mu-c(a-1)(a-2)-(m-2(p-1)) c(a-1)+(m-2(p-1)+\mu) c=0 .
$$

By eliminating $c$ from (5.18) and (5.19), we have

$$
\begin{aligned}
& a^{4}+2(m-2 p-1) a^{3}+\left(m^{2}+4 p^{2}-4 p m-4 m+8 p-2 \mu-1\right) a^{2} \\
& -\left(2 m^{2}+8 p^{2}-8 p m+2 \mu m-4 \mu p-2 \mu-2\right) a+\left(\mu^{2}+2 \mu m-4 \mu p-2 \mu\right)=0,
\end{aligned}
$$

i. e. $\left(a-a^{+}(p-1)\right)\left(a-a^{-}(p-1)\right)\left(a-\left(a^{+}(p-1)+2\right)\right)\left(a-\left(a^{-}(p-1)+2\right)\right)=0$.

In case $a=a^{ \pm}(p-1)$, we have

$$
2 c=-a(a-1)-(m-2 p) a+\mu=2 a^{ \pm}(p-1), \quad \text { i. e. } c=a^{ \pm}(p-1) .
$$

In case $a=a^{ \pm}(p-1)+2$, we have

$$
2 c=2 a^{\mp}(p-1), \quad \text { i. e. } c=a^{\mp}(p-1) .
$$

Therefore, when $a^{+}(p-1) \neq a^{-}(p-1)+2$, i. e. $\nu(p-1) \neq 1,(5.8)=0$ and $(5.10)=0$ if and only if

$$
\sum_{\jmath(c e, p-1)} g_{j(c e, p-1)} \tilde{d} \omega_{j(c e, p-1)}+\sum_{\jmath(e, p-1)} f_{\jmath(e, p-1)} d r \wedge \omega_{j(e, p-1)}
$$

can be written as a convergent sum of forms of types; $\omega^{ \pm}$,

$$
r^{a+(p-1)+2} \tilde{d} \omega+a^{-}(p-1) r^{a+(p-1)+1} d r \wedge \omega
$$

and

$$
r^{a^{-}(p-1)+2} \tilde{d} \omega+a^{+}(p-1) r^{a^{-}(p-1)+1} d r \wedge \omega,
$$

where $\omega$ is a co-exact eigenform of $\tilde{\Delta}$ with eigenvalue $\mu$. If we set $\rho=\tilde{d} \omega$, (5.21) and (5.22) induce $\rho^{ \pm}$; see (3.3). Suppose $a^{+}(p-1)=a^{-}(p-1)+2$, i. e. $\nu(p-1)=1$ which means that $2 p=m$ and $\mu=3 / 4,2 p=m+1$ and $\mu=1$ or $2 p=$ $m+2$ and $\mu=3 / 4$. Then, $(5.8)=0$ and $(5.10)=0$ if and only if (5.20) can be written as a convergent sum of forms of types; $\omega^{ \pm},(5.21)$ and

$$
r^{a^{-}(p-1)+2} \log r \tilde{d} \omega+\frac{1}{2}\left\{\left(a^{-}(p-1)+2\right)^{2}+\mu\right\} r^{a^{-}(p-1)+1} \log r \cdot d r \wedge \omega .
$$

Thus the proof of Proposition 3.1 is complete.

Next, by (5.2), we know that the form $\theta$ satisfying $\Delta \theta=0$ is closed if and only if $\theta$ can be written as a convergent sum of forms of types; $\phi^{+}(\mu=0$ and $\alpha_{p} \leqq 0$, i. e. $\mu=0$ and $\left.2 p \leqq m-1\right), \phi^{-}\left(\mu=0\right.$ and $\alpha_{p}>0$, i. e. $\mu=0$ and $\left.2 p \geqq m\right), \omega^{+}$, $\omega^{-}, \psi^{+}$and $\psi^{-}$. By (5.3), we know that the form $\theta$ satisfying $\Delta \theta=0$ is co-closed 
if and only if $\theta$ can be written as a convergent sum of forms of types; $\phi^{+}, \phi^{-}$, $\omega^{+}, \omega^{-}, \phi^{+}\left(\mu=0\right.$ and $\alpha_{p-2} \geqq 0$, i. e. $\mu=0$ and $\left.2 p \geqq m+3\right)$ and $\psi^{-}\left(\mu=0\right.$ and $\alpha_{p-2}<0$, i. e. $\mu=0$ and $2 p \leqq m+2)$. Thus the proof of Corollary 3.2 is complete.

Finally, we will prove Corollary 3.3. For a $p$-form $r^{a} \phi$, where $a$ is a real number and $\phi$ is the natural extension of a non-zero $p$-form on $N$, we have $\left\|r^{a} \phi\right\|_{0.0,1}^{2}=\int_{0}^{1}\left\|r^{a} \phi\right\|_{(r, r)}^{2} d r=\left(\int_{0}^{1} r^{m-2 p+2 a} d r\right) \cdot\|\phi\|_{0 ; N}^{2}$; see (1.5). Therefore, such a $p$ form $r^{a} \phi$ belongs to $\Lambda^{p ; 0}\left(C_{0,1}(N)\right)$ if and only if $m-2 p+2 a>-1$. Thus, for a $p$-form $r^{a} \phi+r^{b} d r \wedge \phi$, where $\phi$ and $\phi$ are natural extensions of non-zero $p$ and $(p-1)$-forms on $N$, respectively, and $a$ and $b$ are real numbers, $r^{a} \phi+r^{b} d r \wedge \phi$ belongs to $A^{p ; 0}\left(C_{0,1}(N)\right)$ if and only if $a>\alpha_{p-1}$ and $b>\alpha_{p-2}$. Therefore, the form $\theta$ which satisfies $\Delta \theta=0$ and belongs to $\Lambda^{* ; 0}\left(C_{0,1}(N)\right)$ can be written as a convergent sum of forms of types; $\phi^{+}, \phi^{-}(\nu(p)<1), \omega^{+}, \rho^{+}, \rho^{-}(\nu(p-1)<2), \phi^{+}$ and $\psi^{-}(\nu(p-2)<1)$. This, combined with Corollary 3.2, implies Corollary 3.3.

\section{REFERENCES}

[1] Aтіуан, M.F.; Elliptic operators, discrete groups and von Neumann algebras, Soc. Math. de France, Astérisque, 32, 33 (1976), 43-72.

[2] Atiyah, M.F., Patodi, V.K. And Singer, I.M., Spectral asymmetry and Riemannian geometry I, Proc. Camb. Phil. Soc., 77 (1975), 43-69.

[3] Cheeger, J.; Analytic torsion and the heat equation, Ann. of Math. 109(1979), 259-322.

[4] Cheeger, J.; On the spectral geometry of spaces with cone-like singularities, Proc. Nat1. Acad. Sci., U.S.A., 76 No5 (1979), 2103-2106.

[5] De Rham, G.; Variétés Differentiables, Harmann, Paris (1960).

[6] Dodziuk, J.; De Rham Hodge theory for $L^{2}$-cohomology of infinite coverings, Topology, 16 (1977), 157-165.

[7] Dodziuk, AND PATOdI, V.K.; Riemannian structures and triangulations of manifolds, J. of Indian Math. Soc., 40 (1976), 1-52.

[8] Gaffney, M.; The harmonic operator for exterior differential forms, Proc. Natl. Acad. Sci., U.S.A., 37 (1951), 48-50.

[9] Muller, W.; Analytic torsion and $R$-torsion of Riemannian manifolds, Adv. in Math., 28 (1978), 233-305.

[10] Ray, D. AND Singer, I.M.; R-torsion and the Laplacian on Riemannian manifolds, Adv. in Math., 7 (1971), 145-210.

[11] Whitney, H.; Geometric Integration Theory, Princeton Univ. Press, Princeton (1957). 